\title{
A dynamical model for FR II type radio sources with terminated jet activity
}

\author{
Elżbieta Kuligowska
}

\begin{abstract}
Astronomical Observatory of the Jagiellonian University, Orla 171 Cracow, Poland
e-mail: elzbieta@oa.uj.edu.pl
\end{abstract}

Received 1 June 2016 / Accepted 9 October 2016

\begin{abstract}
Context. The extension of the KDA analytical model of FR II-type source evolution originally assuming a continuum injection process in the jet-IGM interaction towards a case of the jet's termination is presented and briefly discussed.

Aims. The dynamical evolution of FR II-type sources predicted with this extended model, hereafter referred to as KDA EXT, and its application to the chosen radio sources.

Methods. Following the classical approach based on the source's continuous injection and self-similarity, I propose the effective formulae describing the length and luminosity evolution of the lobes during an absence of the jet flow, and present the resulting diagrams for the characteristics mentioned.

Results. Using an algorithm based on the numerical integration of a modified formula for jet power, the KDA EXT model is fitted to three radio galaxies. Their predicted spectra are then compared to the observed spectra, proving that these fits are better than the best spectral fit provided by the original KDA model of the FR II-type sources dynamical evolution.
\end{abstract}

Key words. galaxies: active - galaxies: evolution - galaxies: jets

\section{Introduction}

The previously elaborated and published analytical models for the dynamics and radio emission properties of FR II-type radio sources (Fanaroff \& Riley 1974) are all based on the "standard model" of Scheuer (1974) and Blandford \& Rees (1974); the model explains these sources as an interaction of twin jets, composed of highly relativistic particles emerging from the active galactic nucleus (AGN), with the external gaseous environment called intergalactic medium (IGM) surrounding a given galaxy or quasar. The jet formation results from the matter's accretion processes on a supermassive black hole with magnetic field and angular momentum. Interaction of the jets with the IGM produces a supersonic shock enabling the acceleration of the external medium particles and the jet material after their transition through the shock which, in turn, inflates the radio lobes or cocoon and is observed as diffuse emission with a typical powerlaw spectrum.

Non-thermal continuum radio emission of the lobes is due to the synchrotron process and to the inverse-Compton scattering of ambient photons of the cosmic microwave background (CMB). The synchrotron radiation arises from ultra-relativistic particles interacting with the magnetic field. According to classical electrodynamics, the life-time of relativistic electrons emitting medium and short radio waves has to be relatively short. The most energetic electrons, preferentially emitting at high radio frequencies and due to the synchrotron losses, lose their energy at the fastest rate. This implies that, in the absence of a constant injection of fresh particles into the lobes, the synchrotron losses must cause a violent steepening of their radio spectra and a rapid dimming of the source's structure.
However, observed radio lobes are stable, so such long-lived structures have to be continuously powered by new particles from the AGN. This scenario forms the basis of all analytical models of powerful radio galaxies and radio-loud quasars (e.g. Begelman \& Cioffi 1989; Falle 1991; Kaiser et al. 1997; Blundell et al. 1999; Manolakou \& Kirk 2002). All of these models, which differ in their description of how the relativistic particles pass from the jets into the lobes and in their treatment of energy losses and particle transport, assume a constant injection of new particles to the lobes during the time at which the source is observed. This is, clearly, a substantial simplification of these models.

Nevertheless, precise observations show several radio structures (of FR II-type) with so-called double-double morphology strongly suggesting a recurrent jet activity in the AGN (cf. Saikia \& Jamrozy 2010) and also show radio spectra of the lobes with a high-frequency slope significantly exceeding the limiting value of $\alpha_{\text {inj }}+0.5$ expected in the continuum-injection (CI) process. Therefore, in this paper I modify the model of Kaiser, DennettThorpe \& Alexander (1997; hereafter KDA model) by introducing one more free parameter in that model, i.e. a time of the jet's termination, $t_{\mathrm{br}}$, and consider changes in the adiabatic expansion of the cocoon (lobes) and in its radio emission at $t_{\mathrm{br}}$.

This approach was already extensively discussed by Kaiser et al. (2000) in their investigation of the evolution of the outer and inner structures of five selected double-double radio galaxies. However, their considerations were qualitative rather than quantitative. The quantitative results given in the above paper were reached under a number of arbitrary assumptions for the values of basic and very sensitive free parameters of the model, fixing the value of the density of the radio core, fixing the value of the exponent of the initial energy distribution of the relativistic 
particles in the jet flow, taking the unique value of the aspect ratio of the cocoon, etc. Our aim is to provide the model that is flexible enough, thus enabling the determination of these parameters by the fit of the extended model to the observational data of a possibly large representative sample of FR II-type radio sources selected within a wide range of linear size, radio power, and redshift.

Therefore, in this paper I propose the effective formula for describing the dynamics and the luminosity evolution of the lobes of FR II-type sources during an absence of the jet inflow. The brief summary of the original KDA model and its proposed extension is given in Sect. 2. The $P_{v}-D$ diagrams, radio spectra, and other characteristics of dynamical evolution of FR II-type radio sources predicted with this extended model are analysed in Sect. 3. The model fits to the observational data for the three example radio galaxies, providing the expected values of their basic physical parameters (the jet power, internal density and pressure, magnetic field strength, the source's age, and duration of the jet flow) are presented in Sect. 4 along with the radio spectra of these sources resulting from the new model. The goodness of the fit of these spectra to the observational data and the corresponding fits arising from the original KDA model are included. The discussion of the results is presented in Sect. 5 .

\section{Applied model and its extension}

\subsection{Brief summary of the original KDA model}

The model applied is based on a detailed dynamical description by Kaiser \& Alexander (1997) combined with the radiative processes analysed by Kaiser et al. (1997) and extensively summarized in Barai \& Wiita (2006). It assumes a power-law radial density distribution (the simplified King 1972, profile) of unperturbed ambient gas surrounding the radio source as $\rho_{\mathrm{a}}(r)=\rho_{0}\left(r / a_{0}\right)^{-\beta}$, where $\rho_{\mathrm{a}}$ is the ambient density at distance $r$ from the centre of the host galaxy, $\rho_{0}$ is the central density of the radio core with radius $a_{0}$, and $\beta$ is exponent of the density profile. From the energy conservation conditions arises the expression for the total length of the jet at a given age $t$ as

$r_{\mathrm{j}}(t)=c_{1}\left(\frac{Q_{\mathrm{j}}}{\rho_{0} a_{0}^{\beta}}\right)^{1 /(5-\beta)} t^{3 /(5-\beta)}$,

where $Q_{\mathrm{j}}$ is the jet's power and $r_{\mathrm{j}}$ is identified with one-half of the source's linear size $D, r_{\mathrm{j}}=D / 2$. If two of the model parameters, $Q_{\mathrm{j}}$ and $\rho_{0} a_{0}^{\beta}$, are specified, the model predicts the time evolution of the source (its size $D$ and volume of the cocoon, $V$ ) only.

The independent relation between the above parameters is available from a consideration of expected radio emission of the source (its lobes or cocoon) under influence of different energy losses. The energy loss of an electron with the Lorentz factor $\gamma$ is

$\frac{\mathrm{d} \gamma}{\mathrm{d} t}=-\frac{a_{1}}{3} \frac{\gamma}{t}-\frac{4}{3} \frac{\sigma_{\mathrm{T}}}{m_{\mathrm{e}} c} \gamma^{2}\left(u_{\mathrm{B}}+i C\right)$,

where the first term on the right-hand side refers to the adiabatic expansion loss in the source's volume evolving as $V(t) \propto t^{a_{1}}$ and the second term to the combined energy loss due to the synchrotron radiation and inverse-Compton scattering of the CMB radiation; $u_{\mathrm{B}}$ and $i C$ are the energy density of the magnetic field and CMB photons, $a_{1}=(4+\beta) / \Gamma_{\mathrm{c}}(5-\beta)$. Moreover, this model assumes a constant rate at which relativistic particles in the jets are transported from the AGN to the hot spot area interpreted as the region of the particles' reacceleration. The initial energy distribution of injected particles is taken as $n\left(\gamma_{\mathrm{i}}\right)=n_{0} \gamma_{\mathrm{i}}^{-p}$, where $p$ is constant. Minimum energy arguments give the ratio $r$ of the magnetic field's energy density to the sum of the energy densities of the relativistic, $u_{\mathrm{e}}$, and thermal, $u_{\mathrm{T}}$, particles: $r=u_{\mathrm{B}} /\left(u_{\mathrm{e}}+u_{\mathrm{T}}\right)=u_{\mathrm{B}} /\left[u_{\mathrm{e}}\left(1+k^{\prime}\right)\right]=(1+p) / 4$.

Finally, the radio emission of the source (cocoon) at a given frequency is calculated by splitting it into infinitesimal evolving volume elements, i.e. undergoing the adiabatic and radiative losses (cf. Eq. (2)). The sum of the contributions from all of these elements gives the total emission (at a frequency $v$ ), $P_{v}(t)$, as a complicated integral over injection time $t_{\mathrm{i}}$

$$
\begin{aligned}
P_{v}(t)= & \int_{t_{\min }}^{t} \mathrm{~d} t_{\mathrm{i}} \frac{\sigma_{\mathrm{T}} c r}{6 \pi v(r+1)} Q_{\mathrm{j}} n_{0}\left(P_{\mathrm{hc}}\right)^{\left(1-\Gamma_{\mathrm{c}}\right) / \Gamma_{\mathrm{c}}} \\
& \times \frac{\gamma^{3-p} t_{\mathrm{i}}^{a_{1} / 3(p-2)}}{\left.\left[t^{-a_{1} / 3}-a_{2}\left(t, t_{\mathrm{i}}\right) \gamma\right)\right]^{2-p}}\left(\frac{t}{t_{\mathrm{i}}}\right)^{-a_{1}\left(1 / 3+\Gamma_{\mathrm{B}}\right)},
\end{aligned}
$$

where $\left(P_{\mathrm{hc}}\right)$, the ratio of the jet head pressure $\left(p_{\mathrm{h}}\right)$ and the uniform cocoon pressure $\left(p_{\mathrm{c}}\right)$, is a function of $\left(R_{\mathrm{T}}\right)$ - the axial ratio of the cocoon described by the empirical formula adopted from Kaiser (2000); $\Gamma_{\mathrm{c}}$ and $\Gamma_{\mathrm{B}}$ are the adiabatic indices in the equation of state of the cocoon material and the magnetic field, respectively.

The integration of Eq. (3) is performable using a numerical calculation only.

\subsection{Extension of the KDA model}

\subsubsection{Adiabatic expansion of the cocoon after nuclear activity ceases}

The information about termination of the energy supply propagates from the AGN to the radio lobes with the speed of sound. There are arguments for a relatively low internal sound speed in the lobes, for example if there is a significant mixing of the lobe material with the surrounding gaseous environment (e.g. Kaiser et al. 2000). It implies that after switching off the jets, the adiabatic evolution of the lobes of old and large sources may be the same as it was before cease of the nuclear activity for a long time. However, for small and overpressured (with respect to the external medium) lobes, the internal sound speed can be high and their adiabatic evolution can slow down in a relatively short time.

After switching off the supply of new particles to the cocoon, it may still be overpressured with respect to the external gaseous environment and therefore be continuing its expansion behind a bow shock. Such adiabatic evolution of a spherical source (early evolution of a supernova remnant) during the so-called coasting phase was analysed by Kaiser \& Cotter (2002). Although the cocoons of radio sources and the related bow shocks are not spherical, they assume a spherical bow shock with the radius $R_{\mathrm{S}}$ growing with time as $R_{\mathrm{S}} \propto t^{2 /(5-\beta)}$. For simplicity they studied the scenario where a radio source reaches pressure equilibrium with the external medium immediately after the jet switches off and assumed an instantaneous transition between the active and coasting phases. With these assumptions they solved the equation of state for the spherical approximation of the source resulting in steady-state similarity solutions from which we can identify their source's radius $R_{\mathrm{c}}$ with the lobe length $D / 2$ in the 
KDA model. After some transformations, the effective formula for the cocoon (the source) length, describing its adiabatic evolution before and after termination of the jet's activity, is given by

$D\left(t, t_{\mathrm{br}}\right)=\left\{\begin{array}{cl}c_{1}\left(\frac{Q_{\mathrm{j}}}{\rho_{0} d_{0}^{\beta}}\right)^{1 /(5-\beta)} t^{3 /(5-\beta)} & \text { for } t<t_{\mathrm{br}} \\ D\left(t_{\mathrm{br}}\right)\left(\frac{t}{t_{\mathrm{br}}}\right)^{\frac{2\left(\Gamma_{\mathrm{c}}+1\right)}{\Gamma_{\mathrm{c}}\left(7+3 \Gamma_{\mathrm{c}}-2 \beta\right)}} & \text { for } t \geq t_{\mathrm{br}}\end{array}\right.$

where $D\left(t_{\mathrm{br}}\right)$ is the cocoon length at the time of switching off the energy supply. Hereafter we follow the Kaiser \& Cotter approach in the limiting case when the internal sound speed is fast (their model B).

\subsubsection{Analytical formula for the integration of radio power}

The energy loss process due to the synchrotron and inverseCompton scattering of the CMB photons (second term on the right-hand side in Eq. (2)) is characterized by the energy break in the energy spectrum comprising particles with different energies. Expressing the energy densities in Eq. (2), $u_{\mathrm{B}}$ and $u_{\mathrm{iC}}$, by the corresponding magnetic field strengths $B$ and $B_{\mathrm{iC}}$ and the constant coefficient by the relevant constant defined by Pacholczyk (1970), one has

$$
\frac{\mathrm{d} \gamma}{\mathrm{d} t}=-C_{2}\left\{(B \sin \theta)^{2}+B_{\mathrm{iC}}^{2}\right\} \gamma^{2}
$$

where $\theta$ is the pitch angle of the relativistic particles. Integrating Eq. (5) gives the time evolution of the particles' energy $\gamma(t)$ with the energy break $\gamma_{\mathrm{br}}^{-1}=C_{2}\left\{(B \sin \theta)^{2}+B_{\mathrm{iC}}^{2}\right\}^{2} t$ fulfilling the condition $\gamma(t) / \gamma_{\mathrm{br}}<1$. The appearance of $\gamma_{\mathrm{br}}$ causes a break frequency in the observed radio spectrum. If the source is supplied by a constant flow of particles (i.e. CI process), the highest break frequency (for $\theta=90^{\circ}$, i.e. for the particles dominating the emission) is

$v_{\mathrm{br}, \mathrm{CI}}=C_{1} B \gamma_{\mathrm{br}}^{2}=\frac{C_{1}}{C_{2}^{2}} \frac{B}{\left(B^{2}+B_{\mathrm{iC}}^{2}\right)^{2} t^{2}}$

which we assume is valid for $t \leq t_{\mathrm{br}}$, and $C_{1}$ and $C_{2}$ are the physical constants (cf. Pacholczyk 1970). On the other hand, if the pitch angle of the particle distribution is highly isotropic, i.e. all the particles can have any possible pitch angles (the Jaffe-Perola process; hereafter JP), the break frequency valid for $t>t_{\mathrm{br}}$ is

$v_{\mathrm{br}, \mathrm{JP}}=\frac{C_{1}}{C_{2}^{2}} \frac{B}{\left\{\left(2 / 3 B^{2}+B_{\mathrm{iC}}^{2}\right)\left(t-t_{\mathrm{br}}\right)\right\}^{2}}$

where $C_{1} / C_{2}^{2}=2.51422 \times 10^{12}$ and $B_{\mathrm{iC}}=0.318(1+z)^{2} \mathrm{nT}$.

According to the superposition principle the analytical formula for the total radio power of a source (its cocoon) at a given frequency can be rewritten as the sum of two integrals. In the extended model presented here, the first integral gives the source power calculated until the time of the jet termination, $t_{\mathrm{br}}$, while the second adds the radio power emitted from $t_{\mathrm{br}}$ until the actual age of the source, $t$. Therefore, the total power of a source can be written as

$$
P_{v}(t)= \begin{cases}P_{v}\left(t_{\min }, t_{\mathrm{br}}\right)+P_{v}\left(t_{\mathrm{br}}, t\right) & \text { for } t_{\mathrm{br}}>t_{\mathrm{min}} \\ P_{v}\left(t_{\min }, t\right) & \text { for } t_{\mathrm{br}} \leq t_{\min }\end{cases}
$$

In the above equation the first term corresponds to the integral given by Eq. (3) in Sect. 2.1, where the upper limit of integration is changed from $t_{\min }$ to $t_{\mathrm{br}}$. The second term is given by

$P_{v}\left(t_{\mathrm{br}}, t\right)=\frac{\sigma_{T} c}{6 \pi v} \frac{r}{r+1} Q_{\mathrm{j}} P_{\mathrm{hc}}^{\frac{\left(1-\Gamma_{\mathrm{c}}\right)}{\Gamma_{\mathrm{c}}}} \int_{t_{*}}^{t} G\left(t_{\mathrm{i}}\right) H\left(t_{\mathrm{i}}\right) \mathrm{d} t_{i}$,

where $t_{*}=t_{\mathrm{br}}$ if $t_{\mathrm{br}}>t_{\min }$ and $t_{*}=t_{\min }$ if $t_{\mathrm{br}} \leq t_{\mathrm{min}}$,

$\begin{aligned} & H\left(t_{\mathrm{i}}\right)= n_{0}\left(t_{\mathrm{i}}\right) \frac{\gamma^{3-p} t_{\mathrm{i}}^{a_{1} / 3(p-2)}}{\left\{t^{-a_{1} / 3}-a_{2}\left(t, t_{\mathrm{i}}\right) \gamma\right\}^{2-p}}\left(\frac{t}{t_{\mathrm{i}}}\right)^{-a_{1}\left(1 / 3+\Gamma_{\mathrm{B}}\right)}, \\ & G\left(t_{\mathrm{i}}\right)=\frac{\int_{0}^{1} F_{\mathrm{JP}}(x) x^{-p}(1-x)^{p-2} \mathrm{~d} x}{\int_{0}^{1} F_{\mathrm{CI}}(x) x^{-(p+1)}\left\{1-(1-x)^{p-2}\right\} \mathrm{d} x+\int_{1}^{\infty} F_{\mathrm{JP}}(x) x^{-(p+1)} \mathrm{d} x},\end{aligned}$

and the functions $F_{\mathrm{JP}}=\left(v / v_{\mathrm{br}, \mathrm{JP}} / x^{2}\right)$ and $F_{\mathrm{CI}}=\left(v / v_{\mathrm{br}, \mathrm{CI}} / x^{2}\right)$ are the "kernel" synchrotron spectrum of a single ultra-relativistic particle. It should be noted that transient values of $v_{\mathrm{br}, \mathrm{JP}}$ and $v_{\mathrm{br}, \mathrm{CI}}$ are functions of $t_{\mathrm{i}}$, i.e. the time of initial acceleration of the radiating particles. The sum of the two integrals in the denominator of the function $G\left(t_{\mathrm{i}}\right)$ results from a different form of these integrals for $v<v_{\mathrm{br}}$ and for $v \geq v_{\mathrm{br}}$. It is calculated at every step of the integration and all of the infinitesimal values of integrated radio power are multiplied by this function.

\section{Predictions of the extended model}

In order to study the influence of the jet termination on the $P_{v}-D$ diagrams and observed radio spectra, a fiducial source with a set of basic model parameters: $\alpha_{\text {inj }}=(p-1) / 2=0.51, \beta=1.5$, $z=0.1, R_{t}=3, k^{\prime}=0$, adiabatic indices in the equation of state: $\Gamma_{\mathrm{c}}=\Gamma_{\mathrm{B}}=5 / 3, a_{0}=10 \mathrm{kpc}, Q_{\mathrm{j}}=10^{38} \mathrm{~W}$ and $\rho_{0}=10^{-22} \mathrm{~kg} \mathrm{~m}^{-3}$ is used.

\subsection{Evolutionary tracks}

Kaiser et al. (1997) presented the $P_{v}-D$ diagrams (Shklovskii 1963) for the original KDA model applied to fiducial FR II-type radio sources with parameters adopted in agreement with the present knowledge. These diagrams, which constitute a powerful tool for investigation of time evolution of radio sources, show the sources decreasing radio luminosity determined at a given frequency, $P_{v}$, as a function of its linear size $D$ enlarging with time. In this section, the analogous evolutionary tracks of the fiducial FR II-type sources, constructed with the new KDA EXT model and with the use of some revised physical parameters of the source (cf. the list given above) are presented.

Figure 1 shows the $P_{178}-D$ diagrams for the fiducial source with three different values of $\alpha_{\text {inj }}=0.51,0.75$, and 1.0 for $t_{\mathrm{br}}=10$ Myr. The dashed and solid lines show its lobes radio power and size evolution with time in two limiting scenarios: a very slow and a fast internal sound speed in the cocoon, respectively (see Sect. 2.2.1). Within the first scenario, the lobe's length is initially still evolving as in the continuum-injection model (the upper expression of Eq. (4)), and after some time it begins to evolve as in the KDA EXT model. In the second scenario, lobes are continuously expanding according to the KDA EXT model and formula described by the bottom expression of Eq. (4). Finally, dotted lines correspond to the pure KDA evolution. 


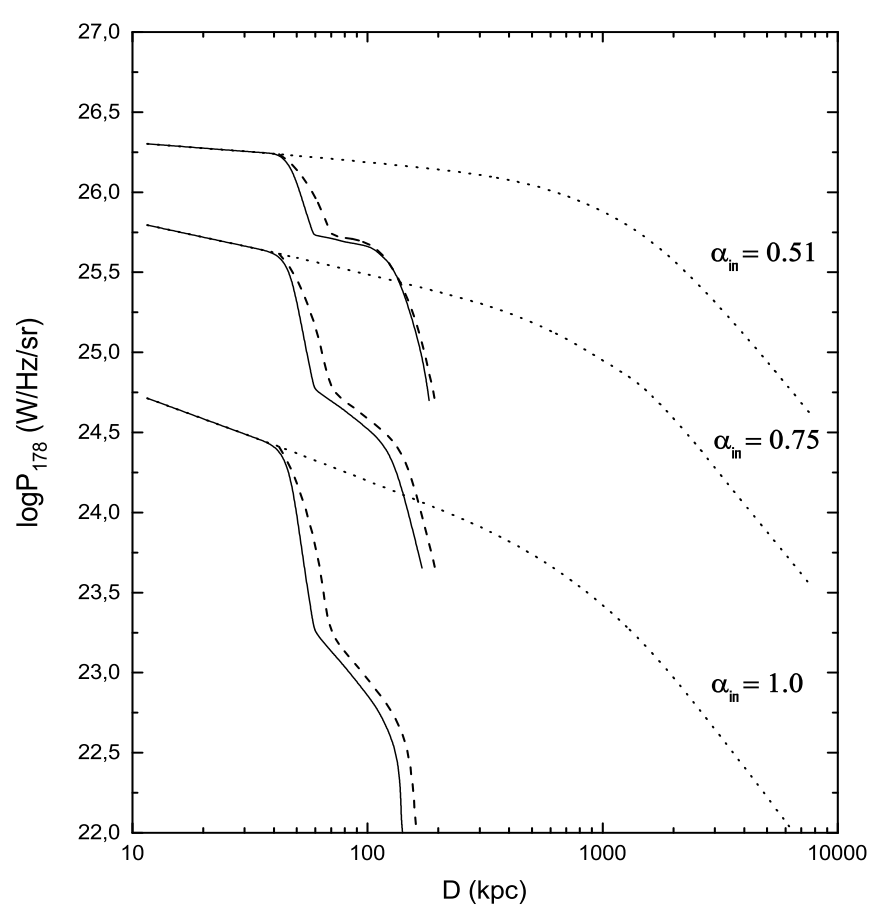

Fig. 1. Comparison of the $P_{178}-D$ diagrams predicted with the KDA and KDAEXT model for the fiducial source with three different values of $\alpha_{\mathrm{inj}}$ and $t_{\mathrm{br}}=10 \mathrm{Myr}$. The solid and dashed lines show predictions of the KDA EXT model for the two limiting cases of the internal sound speed (see the text). The dotted lines show predictions of the pure KDA model. All the KDA EXT diagrams are cut off at the points where their radio powers rapidly diminish and linear sizes tend to a constant value.

These diagrams demonstrate the dependence of various source parameters on its dynamical evolution in the two extreme cases of the internal sound speed (KDA and KDA EXT). The clearly visible breaks may result from the cocoon length evolution (here adopted from Kaiser \& Cotter's limiting case B, in which the internal sound speed is fast - cf. Sect. 2.2.1) is not completely applicable in the case of terminated jet activity. In particular, the information about stopping the jet is not transmitted instantaneously, so the lobes should not begin to expand significantly more slowly immediately after the jet switch-off. It is more probable that its evolution changes smoothly from one regime $(\mathrm{CI})$ to another (KDA EXT).

Since we are not able to provide the right formula for this gradual change, we can only approximate this situation by using the following procedure based on the assumption that the rate of the cocoon growth only changes slowly: the separated model is constructed with the radio luminosity evolving as in the KDA EXT model, but with the lobe length still growing as in the CI regime (KDA) shortly after the jet termination. Then, after the assumed time, the lobes length evolution transforms into the internal sound speed scenario of KDAEXT, but with more recent stopping of the jet activity, which implies using the reduced value of $t_{\mathrm{br}}$ (here equal to the 0.7 of the initial $t_{\mathrm{br}}$ in the KDA EXT). This procedure simulates - very roughly - the slow change described above and is intended to demonstrate that the "broken" KDAEXT plots can be easily smoothed only if the proper formula for the gradual lobe growth can be applied.

It is also worth noting that the abnormal breaks on $P_{178}-D$ diagrams may be the result of numerical effects occurring in the calculation of the lobes radio luminosities. In particular, they may occur because the radio power in the KDAEXT model

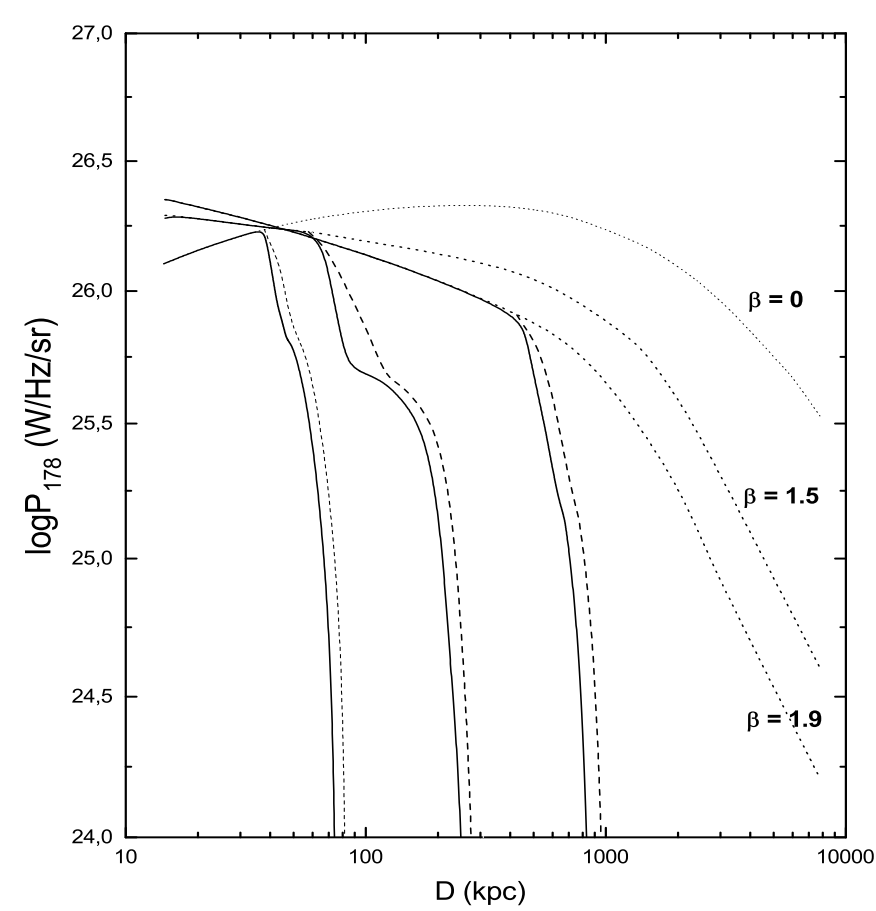

Fig. 2. Comparison of the $P_{178}-D$ diagrams predicted by the KDA and KDA EXT model with different values of $\beta(0,1.5,1.9)$ and $t_{\mathrm{br}}=10$, 20 , and $100 \mathrm{Myr}$, respectively. Calculations are performed for the same set of source parameters as in Fig. 1, but only for $\alpha_{\text {inj }}=0.51$. Solid lines show predictions of the KDA EXT, dotted lines show predictions of the KDA model, while the dashed lines trace the corrected (smoothened) KDAEXT model with the approximated gradual change of the lobe length evolution (see the text).

constitute the sum of three different and independently calculated integrals with the distinct integration limits (cf. Eq. (8)). The characteristic "shoulder" visible in all diagrams corresponds to time $t_{\mathrm{i}}$ at which the integration changes from the case of $t_{\mathrm{br}}>t_{\min }$ to $t_{\mathrm{br}} \leq t_{\min }$. This implies that though we expect the plots to be continuous at this point, some breaks appear as the numerical result of changing the limits of integration and (most likely) more rapid decrease in the first of the integrals in the upper expression of Eq. (8), which cannot keep up with the slower increase in the second integral in this formula and maybe also with the independently evolving lobe length. It is also interesting that these breaks are sharper for the high values of $\alpha_{\text {inj }}$ (cf. Fig. 1)

Figure 2 shows the $P_{178}-D$ diagrams for three different values of $\beta$ : $0,1.5$, and 1.9 and for $\alpha_{\mathrm{inj}}=0.51$ and for $t_{\mathrm{br}}=10$, 20, and $100 \mathrm{Myr}$, respectively. The solid, dashed, and dotted lines correspond to the same models as in Fig. 1. Finally, Fig. 3 present analogous $P_{178}-D$ diagrams for the fiducial source for three different values of $\rho_{0}: 10^{-21}, 10^{-22}$, and $10^{-23} \mathrm{~kg} \mathrm{~m}^{-3}$, with $\alpha_{\mathrm{inj}}=0.51$ and for $t_{\mathrm{br}}=10 \mathrm{Myr}$. Regardless of varying parameters of the fiducial source, evolutionary tracks for the KDA EXT model behave similarly to the ones discussed above, and also here the characteristic plot irregularities can be noticed. It can be then assumed that they are also most probably due to the numerical effects or incorrect formula for calculating the lobe length.

\subsection{Time evolution of the lobe's expansion velocity, internal pressure and the total energy emitted}

The relations between the source's age and the expansion velocity of its lobes, the internal pressure and total energy emitted are 
E. Kuligowska: A dynamical model for FR II type radio sources with terminated jet activity

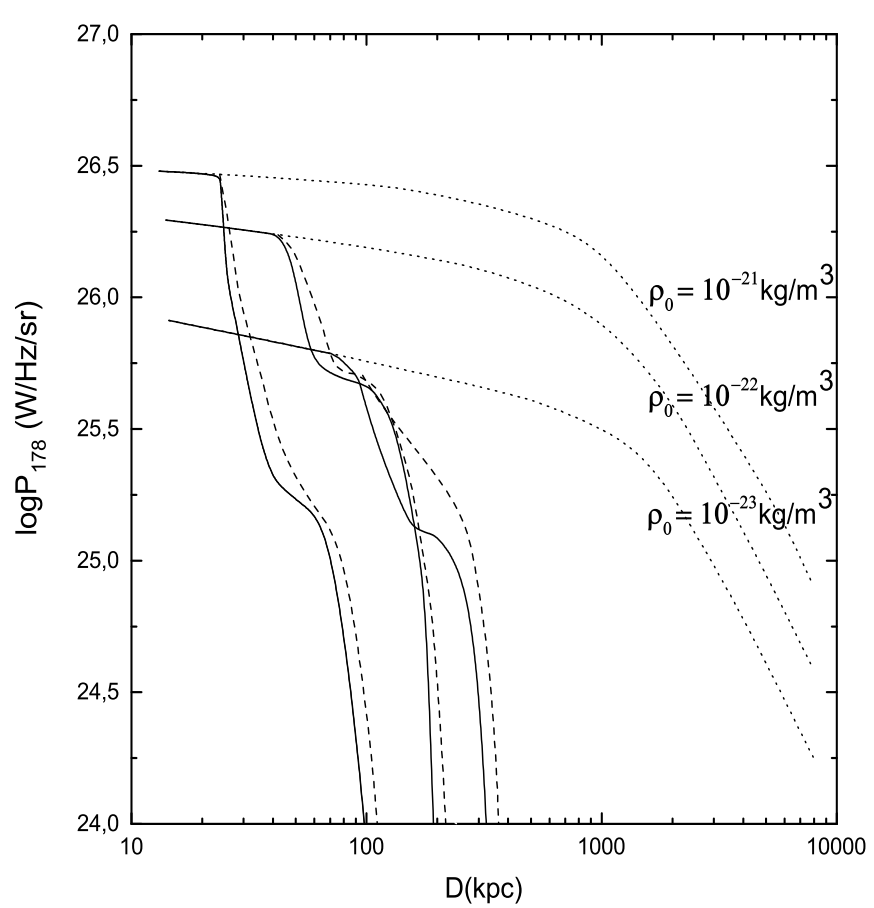

Fig. 3. Comparison of the $P_{178}-D$ diagrams predicted by the KDA and KDA EXT model with different values of $\rho_{0}$ and $t_{\mathrm{br}}=10$ Myr. Calculations are performed for the same set of source's parameters as in Fig. 1 , but only for $\alpha_{\text {inj }}=0.51$. Solid lines show predictions of the KDA EXT, dotted lines show predictions of the KDA model, and the dashed lines trace the same predictions as the analogous lines in the Fig. 2.

also analysed. The instantaneous bow shock velocity $v_{\mathrm{h}}$ is

$v_{\mathrm{h}}(t)=\frac{\partial}{\partial t} D(t)$

and is reduced to the differentiation of Eq. (4) which gives:

$v_{\mathrm{h}}(t)=\left\{\begin{array}{cc}c_{1} \frac{3}{5-\beta}\left(\frac{Q_{j}}{\rho_{0} d_{0}^{\beta}}\right)^{\frac{1}{5-\beta}} t^{\frac{\beta-2}{5-\beta}}, & t<t_{\mathrm{br}} \\ c_{4} D\left(t_{\mathrm{br}}\right) t_{\mathrm{br}}^{-c_{4}} t^{c_{4}-1}, & t \geq t_{\mathrm{br}},\end{array}\right.$

where $c_{4}=\frac{2\left(\Gamma_{\mathrm{c}}+1\right)}{\Gamma_{\mathrm{c}}\left(7+3 \Gamma_{\mathrm{c}}-2 \beta\right)}$.

Figures 4, 5 present the evolution of the lobe's head velocity given as the ratio of the speed of light, $c$, in function of the source's age for the KDA and the KDAEXT model. It is worth noting that the rate of its decrease depends not only on $t_{\mathrm{br}}$ and the $\beta$ exponent, but also on different values of $\Gamma_{\mathrm{c}}$ (for relativistic and cold cocoon material). The diagrams indicate that the process of termination of the nuclear activity strongly affects the rate of the lobe propagation as well. The difference of this rate increases with the increase in the value of the $\beta$ exponent describing the density profile of the external medium.

In the case of a young source, the lobe expansion velocity predicted with the KDA model strongly depends on the density profile of that medium (cf. Fig. 5). For the radio sources with terminated activity (the case of KDA EXT model) this dependence rapidly declines with their growing age because the $\beta$ parameter has a relatively small contribution in the formula describing the lobe's head velocity. On the contrary, this velocity begins to depend more strongly on the value of $\Gamma_{c}$ with the source's age. It can also be seen that for the assumed value of $\beta=0$, the lobe velocities predicted from the models with different values of $t_{\mathrm{br}}$ are

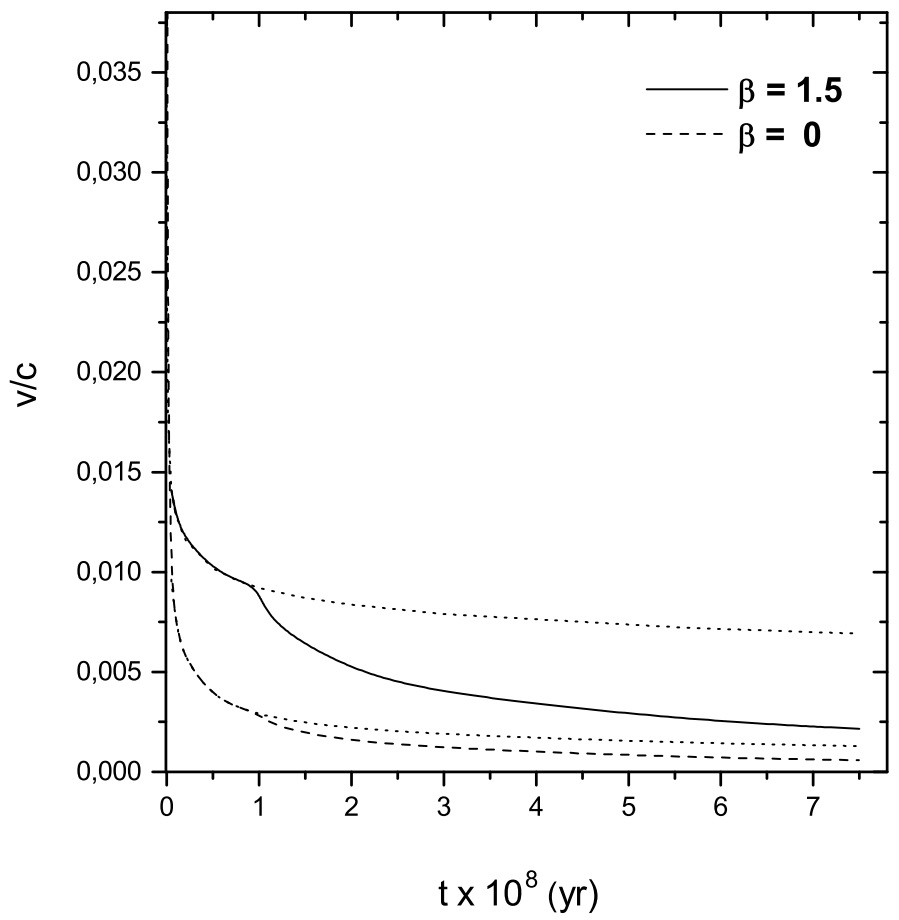

Fig. 4. Velocity of the expanding lobes vs. time. Solid and dashed curves correspond to the KDAEXT and dotted lines follow the KDA model of continuous activity. Parameters are the same as in Fig. 1, but with $t_{\mathrm{br}}=100 \mathrm{Myr}$ and $\Gamma_{\mathrm{c}}=5 / 3$.

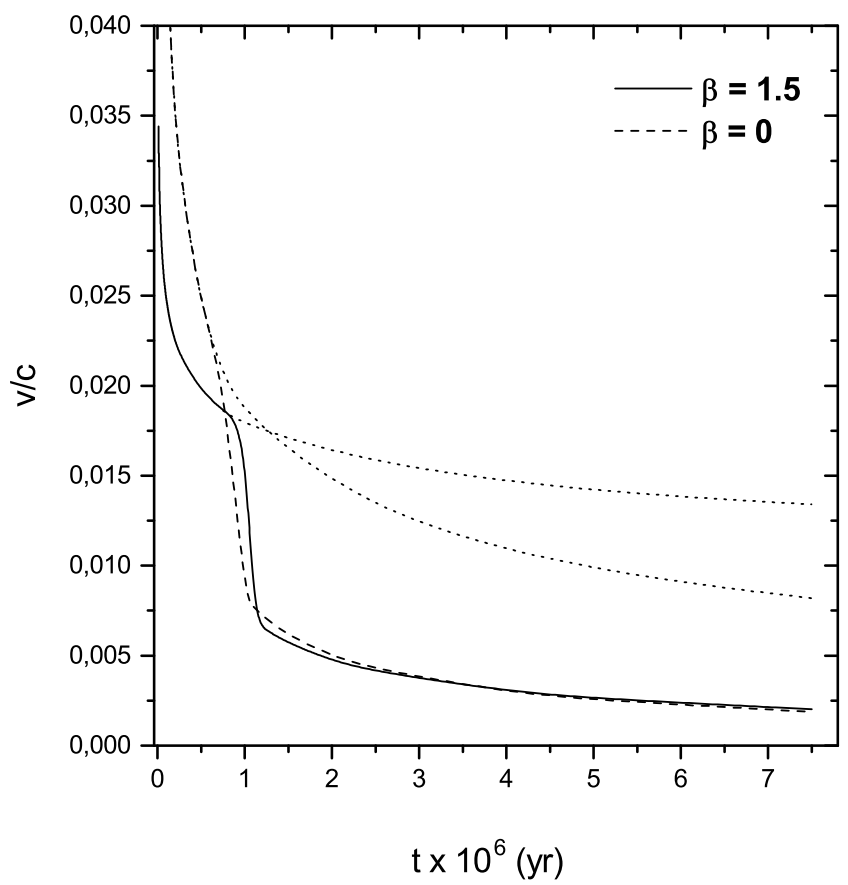

Fig. 5. Velocity of the expanding lobes vs. time. As in Fig. 4, but for $t_{\mathrm{br}}=1 \mathrm{Myr}$.

very similar. The difference between $v$ relations is much more explicit in the case of these two models calculated for $\beta=1.5$.

Following the discussion given by Kaiser \& Cotter for their model B, I also assume that the time evolution of the lobe pressure $p_{\mathrm{c}}$ after switching off the source's nuclear activity, 


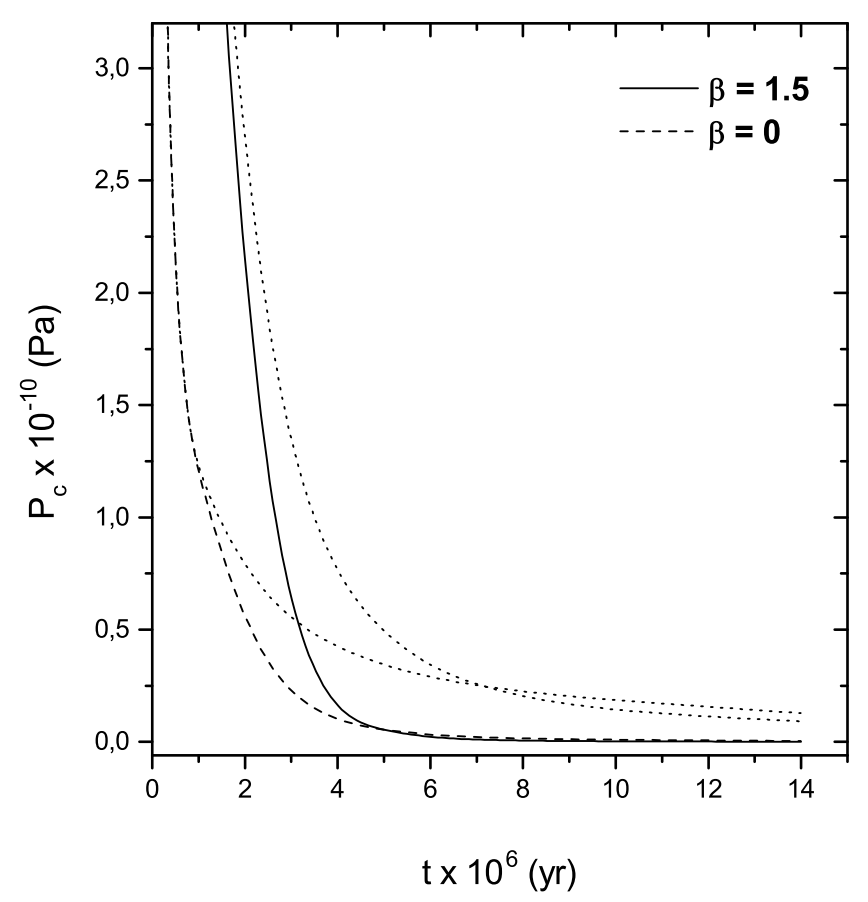

Fig. 6. Internal pressure of the expanding lobes vs. time. Solid and dashed curves correspond to the KDA EXT and dotted lines follow the KDA model of continuous activity. The parameters are the same as in Fig. 1 , with $t_{\mathrm{br}}=1 \mathrm{Myr}$ and $\Gamma_{\mathrm{c}}=5 / 3$.

changes its former decrease according to the formula:

$p_{\mathrm{c}}\left(t>t_{\mathrm{br}}\right)=p_{\mathrm{c}}\left(t_{\mathrm{br}}\right)\left(\frac{t}{t_{\mathrm{br}}}\right)^{-3 \Gamma_{\mathrm{c}} c_{4}}$

Figure 6 presents the behaviour of internal pressure of the expanding lobes due to its growing age. Similarly to the lobe's head velocity, the evolutionary tracks are calculated for both the KDA and KDAEXT models for the two different values of the $\beta$ exponent. In both models the slope of the $p_{\mathrm{c}}(t)$ function is steeper for $\beta=1.5$ than for $\beta=0$, according to Eq. (19). Regardless of that factor, the time evolution of the lobe's internal pressure is faster and much more rapid in the KDA EXT model, which is in good agreement with our physical intuition - in the case of the termination of the jet, the decrease in the lobe's head velocity is expected, as is the gradual decline in that pressure. It is also noteworthy that in the case of the KDA EXT, there is an exponential decay of the internal pressure that, starting at the particular age, is independent of the $\beta$ value. Finally, the evolution of the lobe's internal pressure depends very weakly on the value of $\Gamma_{\mathrm{c}}$.

Figures 7 and 8 show the conversion efficiency of the energy delivered by the jet until its termination into the observed radiation as a function of $t / t_{\mathrm{br}}$. We can expect its decrease owing to the fiducial source's growing age. Similarly to the lobe's head velocity, the evolutionary tracks are calculated for both KDA and KDAEXT models for three different values of the $\beta$ exponent and three values of $R_{\mathrm{T}}$. Both diagrams are presented: $\Gamma_{\mathrm{c}}=4 / 5$ and $\Gamma_{\mathrm{c}}=5 / 3$. The slopes of the efficiency function are steeper for the low values of $\beta$ and $R_{\mathrm{T}}$, and its evolution is much faster for sources with terminated activity while its value is constant during the phase before the jet termination. The value and the further evolution of the lobe's efficiency depends strongly on the value of $\Gamma_{c}$.

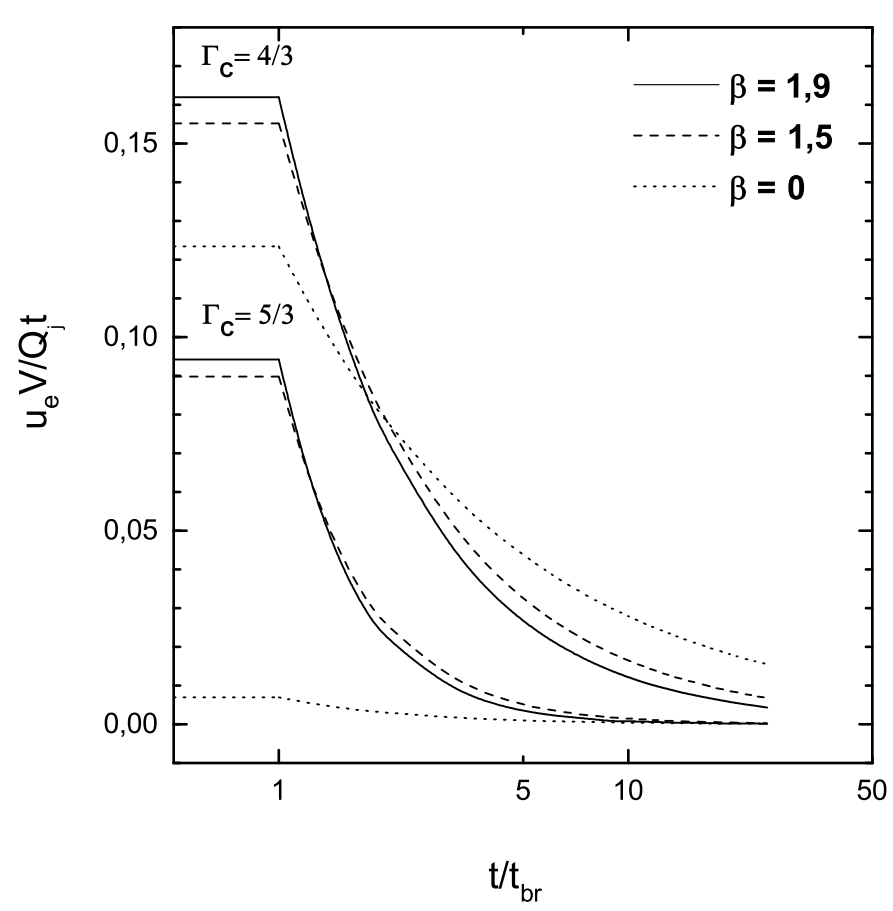

Fig. 7. Total energy of expanding lobes divided by jet power vs. time, calculated for three values of $\beta$, for two general cases: $t / t_{\mathrm{br}}<1$ (KDA model) and $t / t_{\mathrm{br}}>1$ (KDA EXT model). All the upper curves for a given value of $\beta$ are calculated for $\Gamma_{c}=4 / 3$, while the bottom lines for $\Gamma_{\mathrm{c}}=5 / 3$. The other parameters are the same as in Fig. 1 .

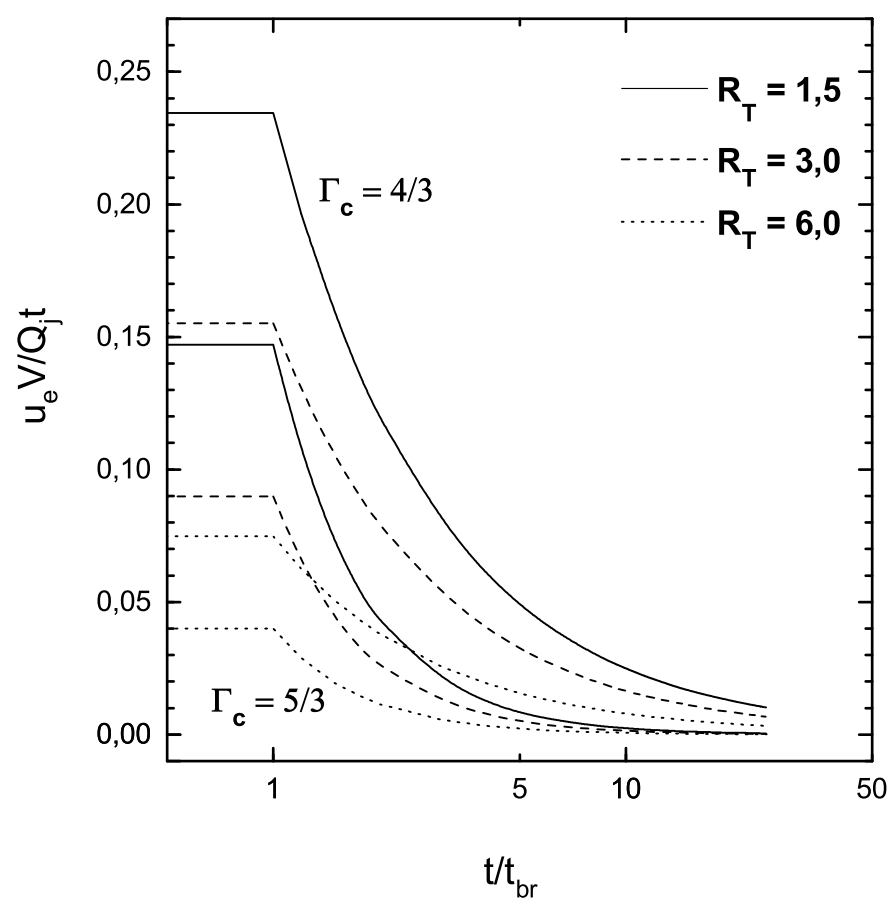

Fig. 8. As in Fig. 7, but for three different values of $R_{T}$.

\subsection{Resulting radio spectra}

Though the $P_{v^{-}} D$ diagrams are very useful in diagnostics of the dynamical evolution of FR II-type radio sources, they suffer from one severe problem - the inability to verify their predictions by direct observations owing to the large timescale of the analysed processes. However, the analytical models of the evolution (including the KDA and KDA EXT) allow the source's luminosity 
E. Kuligowska: A dynamical model for FR II type radio sources with terminated jet activity

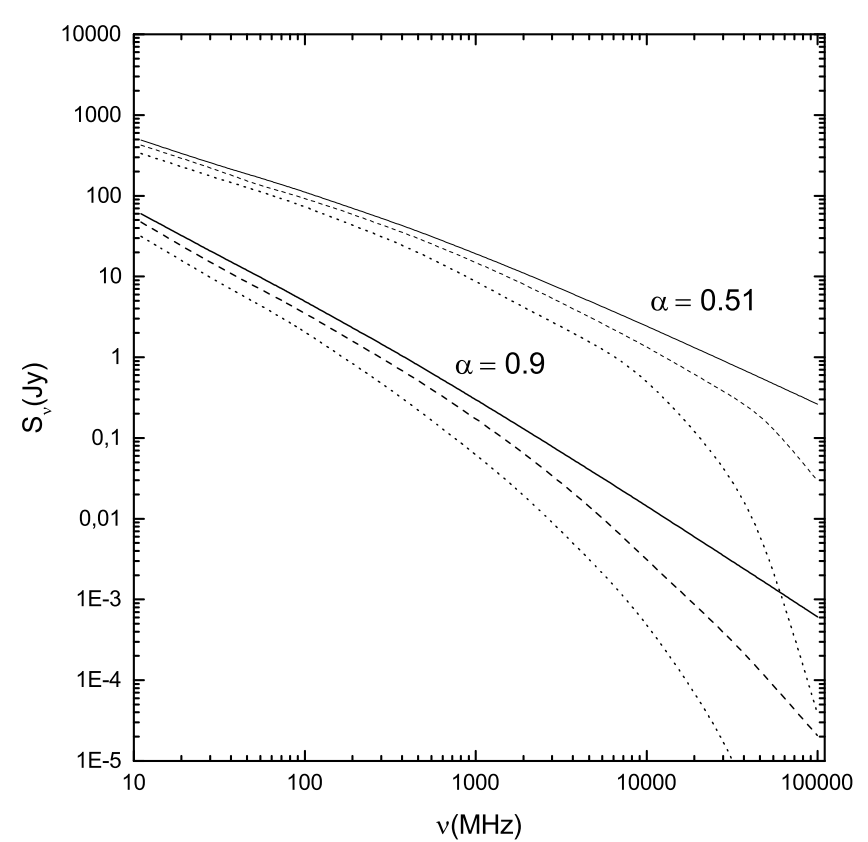

Fig. 9. Comparison of the radio power spectra $\left(P_{v}\right.$ vs. $\left.v\right)$ calculated with the KDA EXT model (dashed and dotted lines for $t_{\mathrm{br}}=33$ and $40 \mathrm{Myr}$, respectively) and the KDA model (solid lines) for the fiducial source with different $\alpha_{\mathrm{inj}}$.

to be predicted at a number of observing frequencies, i.e. its radio spectrum. Here a few examples of such spectra, calculated for different sets of the fiducial source parameters, are shown and compared to the corresponding spectra calculated with the classical KDA model.

Figure 9 presents the radio power spectra $\left(P_{v}\right.$ vs. $\left.v\right)$ of the fiducial source predicted with the KDAEXT model calculated for two different values of $\alpha_{\mathrm{inj}}$, and of two different values of jet switch-off time, $t_{\mathrm{br}}$. It is easy to see that their high-frequency slopes are close to the theoretical synchrotron aging spectrum of Jaffe \& Perola (1973; cf. Fig. 1 in Carilli et al. 1991).

Figure 10 shows the radio power spectra $\left(P_{v}\right.$ vs. $\left.v\right)$ of the fiducial source expected from the KDAEXT model, calculated for two different values of redshift, $z$, as well as two different values of jet switch-off time, $t_{\mathrm{br}}$. It is worth noting that at the high redshift $z=3$, the decrease in radio power is much faster, which is consistent with the cosmological IGM models.

Figures 11 and 12 show radio power spectra $\left(P_{v}\right.$ vs. $\left.v\right)$ of the fiducial source expected from the KDAEXT model and calculated for two different values of $\beta$ and $R_{t}$, respectively. In these cases the spectra are also shown for two values of $t_{\mathrm{br}}$. Additionally, the distinction for $Q_{\mathrm{j}}$ values is introduced in order to ensure that the resulting plots will not be superimposed on each other (because the differences between the spectra of sources with various $\beta$ and $R_{t}$ are relatively low). The general trend indicates that the higher are $Q_{\mathrm{j}}, \beta$, and $R_{t}$, the higher are the values of the radio power, and the spectra resulting from the KDAEXT model are less curved at higher frequencies.

The presented spectra indicate the very strong effect of the jet termination on both linear size and radio luminosity of the lobes, especially for the values of $t_{\mathrm{br}}$ much lower than the actual age of a source, $t$. The characteristic high-frequency break is more rapid in the case of low values of $\beta$ and $\rho_{0}$. The strongest breaking of the spectra due to the rapid decrease in energy also occurs at the high redshift. All the presented spectra show how quickly the lobes of the FR II-type radio source may become invisible (or at

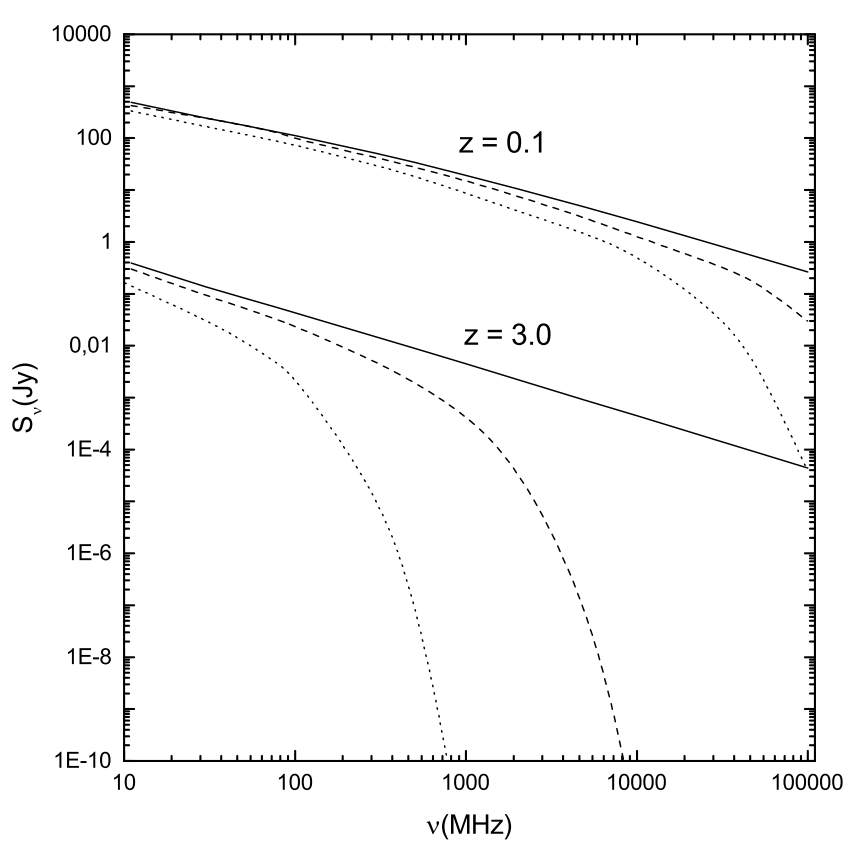

Fig. 10. Comparison of the radio power spectra $\left(P_{v}\right.$ vs. $\left.v\right)$ calculated with the KDAEXT model (dashed and dotted lines for $t_{\mathrm{br}}=33$ and $40 \mathrm{Myr}$, respectively) and the KDA model (solid lines) for the fiducial source with different $z$. We note that the vertical scale is more extended than the other presented spectra in order to show the full shapes of the lower plots for $z=3$.

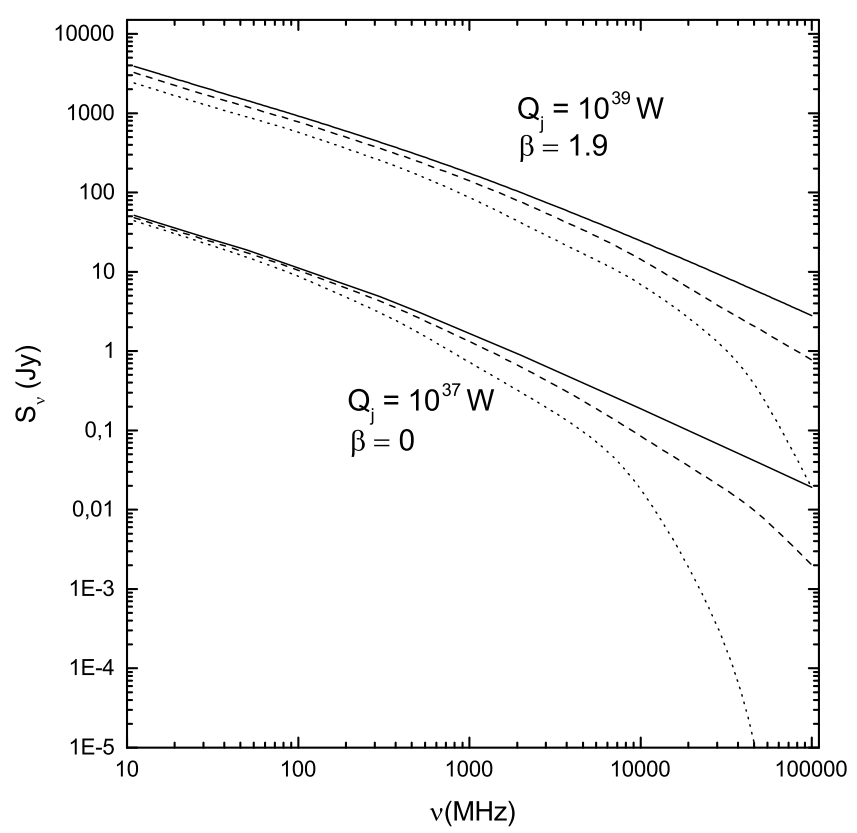

Fig. 11. Comparison of the radio power spectra ( $P_{v}$ vs. $v$ ) calculated with the KDAEXT model (dashed and dotted lines for $t_{\mathrm{br}}=33$ and $40 \mathrm{Myr}$, respectively) and the KDA model (solid lines) for the fiducial source with different $\beta$ and $Q_{\mathrm{j}}$.

least not in the detection range of contemporary radio telescopes) after the time $t=t_{\mathrm{br}}$, only if in the meantime the activity of the nucleus (AGN) has not restarted.

\section{Application of the KDA EXT model}

The KDAEXT model is expected to predict parameters of FR II-type radio sources with the terminated jet activity and 


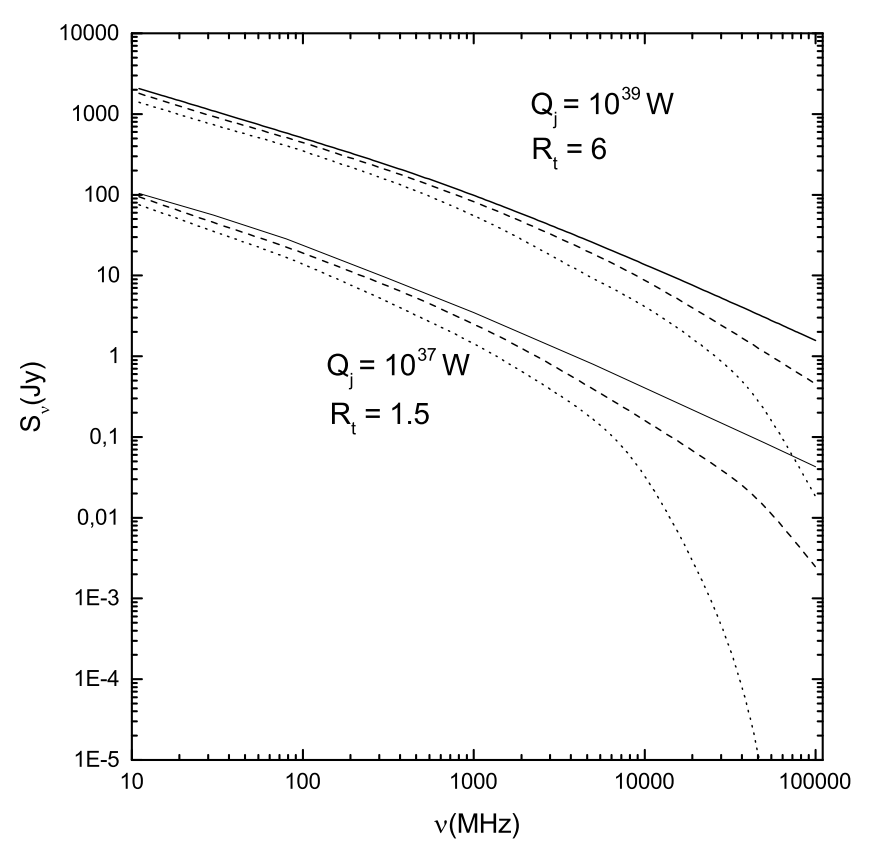

Fig. 12. Comparison of the radio power spectra $\left(P_{v}\right.$ vs. $\left.v\right)$ calculated with the KDAEXT model (dashed and dotted lines for $t_{\mathrm{br}}=33$ and $40 \mathrm{Myr}$, respectively) and the KDA model (solid lines) for the fiducial source with different $R_{t}$ and $Q_{\mathrm{j}}$.

ambient medium conditions for which the KDA model does not give reliable solutions. However, the spectra, sizes, and volumes of real sources are known from observations, and the values of the unknown "free" model parameters are the subject of our interest. The original KDA model, which assumes values for a number of its free parameters, enables the prediction of the time evolution of the source's length $D$, and its radio luminosity $P_{v}$, at a given observing frequency. To solve the reverse problem of determining the values of $t, \alpha_{\text {inj }}, Q_{\text {jet }}$, and $\rho_{0}$ for a real radio source, it is possible to use the DYNAGE algorithm (Machalski et al. 2007) to find these values by a fit to its four observational parameters: size $D$, volume $V$, radio luminosity $P_{v}$ and radio spectrum $\alpha_{v}$, which provides $P_{v, i}$ at a number of observing frequencies $i=1,2,3, \ldots$ This implies that it is also possible to verify the accuracy of the KDAEXT model for a given source with a highly steepened spectrum by deriving its spectra calculated from both the "best age-solution" of the KDA model (DYNAGE) and a similar solution found with the KDA EXT model, and then comparing the results of these two models with the multifrequency observations of this source.

\subsection{Sample of examined radio sources}

Both models have been applied to six radio sources showing a strong bend in their observed radio spectra. This small sample includes three well-known $3 \mathrm{C}$ sources with small linear sizes and (in two cases) relatively high redshift (see Table 1). However, the KDA EXT model is expected to be especially useful in the case of giant FR II sources (i.e. J1428+3938; Machalski et al. 2006; Machalski 2011), which are also examined here. Two radio sources with restarting activity, J1453+3308 (Konar et al. 2006; Machalski et al. 2009) and J1548-3216 (Machalski et al. 2010), are included in the sample as examples of typical DDRG sources for which previous authors studied and modelled their individual lobes. Given the fact that observations of their secondary structure indicates the "young" (renewed) jet emission, interacting and propagating within the previous "old" emission in the same direction, it is interesting to examine how this may affect the "old" primary structure (in particular its dynamical age).

Table 1 presents the observational data of the sources used for the fits. The errors in the flux measurements are taken from the references given under Table 1; however, in some cases great differences can be seen in the error values given in the data bases of the original publications. Moreover, some published errors are evidently underestimated, thus the relevant errors are arbitrariry enlarged, for example the errors claimed by Vigotti et al. (1999).

The "largest angular size" ( $L A S$ ) estimated from radio maps is given in arc seconds. The $R_{t}$ values are adopted from radio maps or the earlier publications. Table 1 also includes the presumed inclination angle of the source's jet axis, $\theta$. The projected linear size of the sources, $D$, is calculated as

$D=4.848 \times 10^{-6} L_{\mathrm{A}}(z) L A S$.

The observed flux densities for the $3 \mathrm{C}$ sources are taken from the NED NASA database. The flux densities for J1428+3938 are derived from various radio surveys (7C, B3-VLA, WENSS, NVSS, Miyun). The flux density data from dedicated observations with the GMRT and VLA arrays for the outer lobes of J1453+3308 and $\mathrm{J} 1548-3216$ are taken from the original publications in order to model their extended, outer FR II-type structures only. Where it was possible, original flux densities were previously corrected by substacting the core emission from the total flux of the sources. The values of the sources' radio power $P_{v}-$ which are the proper physical observables to use in the modelling - are calculated from the given flux densities according to the formula

$P_{v}=S_{v} L_{\mathrm{D}}(z)^{2}(1+z)^{\left(\alpha_{v}-1\right)}$

where $L_{\mathrm{A}}(z)$ (in Eq. (13) and $L_{\mathrm{D}}(z)$ (in Eq. (14)) are the angular and luminosity distances of the source, respectively, determined with the Cosmological Calculator of Wright (2006) assuming a flat Universe with Hubble constant $H_{0}=71 \mathrm{~km} \mathrm{~s}^{-1} \mathrm{Mpc}^{-1}$ and the $\Lambda$ CDM model with cosmological parameters $\Omega_{\mathrm{m}}=0.27$ and $\Omega_{\Lambda}=0.73$, and where $\alpha_{v}$ is the spectral index measured as the spectrum slope gradient calculated separately for every pair of neighbouring radio flux densities.

It is worth noting a need to know the source's radio power $P_{v}$ for at least five observing frequencies for a proper application of the KDA and KDA EXT models. In general, the wider the frequency coverage of the radio spectra of the source, the more precise is the model fit. This indicates that it is also advisable to know the observed flux densities for the very low (about $10-20 \mathrm{MHz}$ ) and very high (above $10 \mathrm{GHz}$ ) radio frequency in order to take it into account in the modelling. However, 18-25 flux density data points covering the frequency range $22 \mathrm{MHz}$ to $15 \mathrm{GHz}$ are provided for the 3C sample sources. For the three remaining sources presented in Table 1 there are at least seven flux densities covering the quite broad frequency range, although some hopes are connected with the new LOFAR interferometer.

\subsection{Fitting procedure}

The fitting method consists of two steps. In the first, the best "age-solution" of the KDA model ( $t_{\mathrm{KDAEXT}}$; Table 8$)$ is determined using the DYNAGE algorithm. However, as expected, the best model fit and resulting spectra remain unsatisfied as long as we are trying to reproduce observed highly steepened spectrum with the model assuming the CI process of the energy supply 
E. Kuligowska: A dynamical model for FR II type radio sources with terminated jet activity

Table 1. Observational data for the selected FR II-type sources with strongly curved spectra.

\begin{tabular}{|c|c|c|c|c|c|c|}
\hline Name & $3 \mathrm{C} 184$ & $3 \mathrm{C} 217$ & $3 \mathrm{C} 438$ & $\mathrm{~J} 1428+3938$ & $\mathrm{~J} 1453+3308$ & J1548-3216 \\
\hline $\bar{z}$ & 0.994 & 0.898 & 0.290 & 0.5 (est) & 0.249 & 0.1082 \\
\hline$L A S\left[{ }^{\prime \prime}\right]$ & 4.8 & 12.1 & 22.4 & 269 & 336 & 522 \\
\hline$R_{t}$ & 3.0 & 4.0 & 2.7 & 3.4 & 3.8 & 2.9 \\
\hline$D[\mathrm{kpc}]$ & 38.5 & 94 & 100 & 1630 & 1297 & 998 \\
\hline$\theta\left[^{\circ}\right]$ & 90 & 70 & 90 & 90 & 90 & 90 \\
\hline$v[\mathrm{MHz}]$ & & & $S_{v} \pm \Delta S_{v}[\mathrm{mJy}]$ & & & \\
\hline 22 & $61000 \pm 5000^{14}$ & & & & & \\
\hline 26 & & $43000 \pm 6000^{8}$ & $228000 \pm 12000^{8}$ & & & \\
\hline 38 & $37200 \pm 3720^{7}$ & $39000 \pm 5850^{9}$ & $150290 \pm 40650^{19}$ & & & \\
\hline 38 & & & $162840 \pm 48850^{8}$ & & & \\
\hline 74 & $23660 \pm 2370^{10}$ & $25380 \pm 25^{10}$ & $81560 \pm 8160^{10}$ & & & \\
\hline 86 & $23800 \pm 2700^{8}$ & $24400 \pm 1300^{8}$ & $86700 \pm 8720^{8}$ & & & \\
\hline 151 & $14870 \pm 893^{7}$ & $16498 \pm 294^{21}$ & & $971 \pm 109^{4}$ & $2165 \pm 110$ & \\
\hline 151 & & & & $990 \pm 95^{7}$ & & \\
\hline 160 & & & & & & $8400 \pm 840$ \\
\hline 178 & $14652 \pm 700^{9}$ & $15590 \pm 600^{20}$ & $51400 \pm 4100^{19}$ & & $2020 \pm 200$ & \\
\hline 178 & $14388 \pm 719^{8}$ & & $49620 \pm 2300^{19}$ & & & \\
\hline 232 & & & & $810 \pm 100^{3}$ & & \\
\hline 240 & & & & & $1667 \pm 250$ & \\
\hline 325 & $9676 \pm 350^{1}$ & $8970 \pm 356^{1}$ & $29940 \pm 1080^{1}$ & $428 \pm 34^{1}$ & $1365 \pm 140$ & \\
\hline 334 & & & & & $1456 \pm 112$ & $4737 \pm 710$ \\
\hline 365 & $9083 \pm 328^{11}$ & $8372 \pm 242^{11}$ & $26400 \pm 950^{11}$ & & & \\
\hline 408 & & $7840 \pm 610^{20}$ & $23760 \pm 480^{15}$ & & & \\
\hline 408 & & $7090 \pm 280^{6}$ & $25180 \pm 1980^{19}$ & $270 \pm 31^{6}$ & & \\
\hline 605 & & & & & $970 \pm 75$ & \\
\hline 619 & & & & & & $3141 \pm 252$ \\
\hline 750 & $4230 \pm 177^{8}$ & $4060 \pm 208^{8}$ & $13700 \pm 700^{19}$ & & & \\
\hline 750 & $4200 \pm 200^{9}$ & $4300 \pm 140^{20}$ & & & & \\
\hline 1287 & & & & & $442 \pm 34$ & \\
\hline 1384 & & & & & & $1733 \pm 87$ \\
\hline 1400 & $2582 \pm 78^{2}$ & $2087 \pm 63^{2}$ & $6854 \pm 220^{2}$ & $83 \pm 4^{2}$ & $426 \pm 36$ & \\
\hline 1400 & $2275 \pm 170^{18}$ & $2230 \pm 70^{20}$ & $6940 \pm 220^{19}$ & & & \\
\hline 1477 & & & $6410 \pm 128^{16}$ & & & \\
\hline 2495 & & & & & & $963 \pm 30$ \\
\hline 2695 & $1183 \pm 60^{8}$ & $1011 \pm 80^{20}$ & $3260 \pm 155^{19}$ & & & \\
\hline 2700 & & $1020 \pm 50^{20}$ & $3300 \pm 160^{19}$ & & & \\
\hline 4830 & & & $1635 \pm 164^{17}$ & & & \\
\hline 4850 & $618 \pm 455^{12}$ & $550 \pm 49^{12}$ & $1607 \pm 143^{18}$ & & & \\
\hline 4860 & & & & $13 \pm 3^{5}$ & $104 \pm 8$ & $415 \pm 42$ \\
\hline 4900 & & & $1580 \pm 60^{19}$ & & & \\
\hline 5000 & $596 \pm 40^{20}$ & $477 \pm 60^{8}$ & $1529 \pm 60^{19}$ & & & \\
\hline 8440 & & & $765 \pm 38^{13}$ & & & \\
\hline 10550 & & & & $3 \pm 2^{6}$ & & \\
\hline 10695 & & $122 \pm 38^{8}$ & $640 \pm 40^{19}$ & & & \\
\hline 10705 & $216 \pm 30^{8}$ & $130 \pm 40^{9}$ & $600 \pm 38^{8}$ & & & \\
\hline 14900 & $150 \pm 20^{8}$ & $120 \pm 20^{8}$ & $390 \pm 30^{8}$ & & & \\
\hline
\end{tabular}

References. ${ }^{(1)}$ WENSS (Rengelink et al. 1997); (2) NVSS (Condon et al. 1998); ${ }^{(3)}$ Miyun (Zhang et al. 1997); (4) 7C (Waldram et al. 1996); ${ }^{(5)}$ Machalski et al. (2006); (6) B3-VLA (Vigotti et al. 1999); ${ }^{(7)}$ 6CII (Hales et al. 1988); ${ }^{(8)}$ Laing \& Peacock (1980); ${ }^{(9)}$ Kellermann \& Pauliny-Toth (1973); ${ }^{(10)}$ VLSS (Cohen et al. 2007); (11) TXS (Douglas et al. 1996); ${ }^{(12)}$ GB6 (Gregory et al. 1996); ${ }^{(13)}$ Hardcastle et al. (1998); ${ }^{(14)}$ Roger et al. (1986); (15) B3 (Ficarra et al. 1985); (16) Leahy \& Pearley (1991); ${ }^{(17)}$ Griffith et al. (1991); ${ }^{(18)}$ White \& Becker (1992); ${ }^{(19)}$ Kühr et al. (1981); ${ }^{(20)} \mathrm{Kühr}$ et al. (1979); ${ }^{(21)} 7 \mathrm{CN}$ (Riley et al. 1999). The flux densities for J1453+3308 and J1548-3216 are adopted from Konar et al. (2006) and Machalski et al. (2010), respectively. 
to the lobes (the case of the KDA model). To solve this problem, I performed the KDA fits using only the low-frequency parts of the flux density data, i.e. rejecting the extremely highfrequency fluxes that increase the curvature of the source's observed spectra.

Following the original KDA analysis, their Case 3 where both the cocoon material and the ambient medium are assumed to be described by the "cold" equation of state (i.e. $\Gamma_{\mathrm{c}}=\Gamma_{\mathrm{a}}=$ $\left.\Gamma_{\mathrm{B}}=5 / 3\right)$ is adopted. The equipartition condition for the initial ratio of the energy densities between the source's magnetic field and relativistic particles, $r \equiv u_{\mathrm{B}} / u_{\mathrm{e}}=(1+p) / 4$, is also assumed, which is well supported by the X-ray observations of the lobes in powerful radio sources (Kataoka \& Stawarz 2005). Finally, following Daly (1995) and Blundell et al. (1999), I adopt $\beta=1.5$ for all of the examined sources. The remaining model free parameters are the same as for the fiducial source in Sect. 3 . The final result of this step is the determination of four model parameters: the initial particle-energy distribution described by $\alpha_{\text {inj }}$; the fitted age, $t$; the corresponding values of the jet power, $Q_{\mathrm{j}}$; and the central core density, $\rho_{0}$.

In the second step, a number of values for the jet switch-off time $t_{\mathrm{br}}$, fulfilling the $t_{\mathrm{br}}<t$ condition, is selected. For each of these values, Eq. (8), i.e. Eqs. (3) and (9) are solved numerically, providing the source power, $P_{v_{e m}}$, at the number of frequencies (in the source frame) corresponding to a given $t_{\mathrm{br}}$ value. The best fit is determined using the least-squares method by minimizing the expression

$\chi_{\text {red }}^{2}=\frac{1}{n-3} \sum_{n}\left(\frac{S_{v_{0}}-S_{\mathrm{MOD}}}{\Delta S_{v_{0}}}\right)^{2}$,

where $S_{v_{0}}$ and $\Delta S_{v_{0}}$ are flux densities and $S_{\text {MOD }}$ are the model flux densities re-calculated from the model values of $P_{v_{e m}}$ according to

$S_{\mathrm{MOD}}=P_{v_{\mathrm{em}}}\left(\frac{1+z}{L_{\mathrm{D}}^{2}(z)}\right)=P_{v_{0}(1+z)}\left(\frac{1+z}{L_{\mathrm{D}}^{2}(z)}\right)$.

The $S_{\text {MOD }}$ flux densities, resulting from the KDA and KDA EXT models, are given in Tables 2-7 and are compared to the observed flux densities which determine radio spectra of the sample sources.

\subsection{Fitting results}

In order to compare the KDA EXT fits with the KDA solutions, and with similar results already published for the sample sources (including those from the KDA model for entire sources or their radio lobes only), the values of $\chi_{\text {red }}^{2}$, determining the goodness of fit, are calculated for all of the best fits of the KDA models and the best fit of the KDA EXT model. The latter fits are characterized by $\alpha_{\text {inj }}$ values that are flatter and dynamical ages that are greater (especially for larger and older sources) than those with the KDA fits.

Table 8 presents values of the model free parameters and some derivative physical parameters of the sample sources derived from their best fit of the KDA and KDA EXT models. These derivative parameters are the cocoon pressure, $p_{\mathrm{c}}$; the total emitted energy, $U_{\mathrm{c}}$; the strength of the magnetic field, $B_{\text {eq }}$; and the radial expansion speed of the cocoon's head, $v_{\mathrm{h}}$. It is worth noting that their values are calculated for $t>t_{\mathrm{br}}$ (after the switch-off). Namely, $p_{\mathrm{c}}$ for $t>t_{\mathrm{br}}$ is calculated with Eq. (12), $U_{\mathrm{c}}$ is given by the formula $U_{\mathrm{c}}(t)=u_{\mathrm{c}}(t) V_{\mathrm{c}}(t)=p_{\mathrm{c}}(t) V_{\mathrm{c}}(t) /\left(\Gamma_{\mathrm{c}}-1\right)$, and $v_{\mathrm{h}}(t)$ with Eq. (11). A compilation of the results obtained for the individual sources from the sample is presented below.
Table 2. Flux densities resulting from the KDA and KDA EXT models for 3C 184 and their goodness of fit to the observed data.

\begin{tabular}{rrrr}
\hline \hline$v_{0}$ & $S_{v_{0}} \pm \Delta S_{v_{0}}$ & $\begin{array}{r}S_{\mathrm{MOD}} \\
\text { KDA }\end{array}$ & $\begin{array}{r}S_{\mathrm{MOD}} \\
\text { KDA EXT }\end{array}$ \\
\hline 22 & $61000 \pm 5000$ & 69177 & 60377 \\
38 & $37200 \pm 3720$ & 46877 & 42388 \\
74 & $23660 \pm 2370$ & 28377 & 26961 \\
86 & $23800 \pm 2700$ & 25407 & 24262 \\
151 & $14870 \pm 893$ & 16179 & 16116 \\
178 & $14652 \pm 700$ & 14124 & 14240 \\
178 & $14388 \pm 719$ & 14124 & 14240 \\
325 & $9676 \pm 350$ & 8472 & 8887 \\
365 & $9083 \pm 328$ & 7655 & 8088 \\
750 & $4230 \pm 177$ & 4018 & 4377 \\
750 & $4200 \pm 200$ & 4018 & 4377 \\
1400 & $2582 \pm 78$ & 2244 & 2456 \\
1400 & $2275 \pm 170$ & 2244 & 2456 \\
2695 & $1183 \pm 60$ & 1191 & 1254 \\
4850 & $618 \pm 55$ & 665 & 643 \\
5000 & $596 \pm 40$ & 646 & 621 \\
10705 & $216 \pm 30$ & 299 & 238 \\
14900 & $150 \pm 30$ & 212 & 146 \\
\hline$\chi_{\text {red }}^{2}$ & & 5.94 & 1.89 \\
\hline & & &
\end{tabular}

\subsection{1. $3 \mathrm{C} 184$}

3C 184 is an example of powerful high-redshift radio galaxies embedded in a cluster environment (Belsole et al. 2004). Its lobe length is rather small and equal to $38.5 \mathrm{kpc}$. It may then be supposed that this source is relatively young. It is worth noting that in the case of 3C 184 (and in two other 3C sources examined in this paper) its radio spectrum is observed within a wide range of frequencies extending from $22 / 26 \mathrm{MHz}$ to even $15 \mathrm{GHz}$.

The spectra resulting from the predicted flux densities $S_{\text {MOD }}$ (KDA) and $S_{\text {MOD }}$ (KDA EXT) are shown in Fig. 13. The KDA EXT spectrum is evidently much more bent than the KDA spectrum and fits better to the flux densities measured at both, low and high frequencies. The goodness of the fit to data points $\left(\chi_{\text {red }}^{2}\right.$ values at the bottom of Table 2$)$ clearly indicate a superiority of the KDA EXT solution over the KDA.

3C 184 is the only source in my sample whose fitted age is less than 1 Myr. However, this young age is accompanied by low relative expansion speed of the lobes' head $v_{\mathrm{h}} / c$, being only about 2-3 times higher than these for the three giant-sized sources in the sample. This is concordent with the "best-fit" age solution with the assumed jet termination. Perhaps it suggest that this high redshift structure is not at an early stage of its evolution, and will not evolve into a structure much larger than $100 \mathrm{kpc}$.

\subsubsection{C 217}

This high-redshift radio galaxy was included in Sample 3 (3CRR FR II-type sources with $z>0.5$ and $D<400 \mathrm{kpc}$ ) in Kuligowska et al. (2009). 3C 217 is another example of a relatively young and small $(D=94 \mathrm{kpc})$ source with a strongly bent radio spectrum.

The spectra resulting from the KDA and KDA EXT models and overlain the data points are shown in Fig. 14. Similarly to 3C 184, the KDA EXT model spectrum is much more bent than 
E. Kuligowska: A dynamical model for FR II type radio sources with terminated jet activity

Table 3. Flux densities resulting from the KDA and KDA EXT models for $3 \mathrm{C} 217$ and their goodness of fit to the observed data.

\begin{tabular}{rrrr}
\hline \hline$v_{0}$ & $S_{v_{0}} \pm \Delta S_{v_{0}}$ & $\begin{array}{r}S_{\mathrm{MOD}} \\
\text { KDA }\end{array}$ & $\begin{array}{r}S_{\mathrm{MOD}} \\
\text { KDA EXT }\end{array}$ \\
\hline 26 & $43000 \pm 6000$ & 57885 & 53440 \\
38 & $39000 \pm 5850$ & 44624 & 42332 \\
74 & $25380 \pm 2590$ & 27200 & 27396 \\
86 & $24400 \pm 1300$ & 24242 & 24688 \\
151 & $16498 \pm 294$ & 15544 & 16227 \\
178 & $15590 \pm 600$ & 13601 & 14287 \\
325 & $8970 \pm 324$ & 8214 & 8989 \\
365 & $8372 \pm 242$ & 7440 & 8168 \\
408 & $7090 \pm 280$ & 6754 & 7398 \\
408 & $7840 \pm 610$ & 6754 & 7398 \\
750 & $4060 \pm 208$ & 3923 & 4127 \\
1400 & $2086 \pm 62$ & 2201 & 2126 \\
1400 & $2230 \pm 70$ & 2201 & 2126 \\
2695 & $1011 \pm 80$ & 1171 & 1038 \\
2700 & $1020 \pm 50$ & 1168 & 1038 \\
4850 & $550 \pm 49$ & 654 & 509 \\
5000 & $477 \pm 60$ & 635 & 487 \\
10695 & $122 \pm 38$ & 294 & 150 \\
10705 & $130 \pm 40$ & 293 & 149 \\
14900 & $120 \pm 20$ & 201 & 84 \\
\hline$\chi_{\text {red }}^{2}$ & & 8.00 & 1.21 \\
\hline & & &
\end{tabular}

that predicted by the KDA model. The $\chi_{\text {red }}^{2}$ values at the bottom of Table 3 clearly indicate a significantly higher goodness of fit in the case of the KDA EXT solution.

\subsection{3. $3 \mathrm{C} 438$}

Similarly to 3C $217,3 \mathrm{C} 438$ is a typical but low-redshift FR IItype source with linear size not exceeding $100 \mathrm{Mpc}$. Also, its observed radio spectrum shows considerable bending from the middle frequencies to the higher ones. The spectra predicted with the KDA and KDAEXT models are shown in Fig. 15. Again, the relevant $\chi_{\text {red }}^{2}$ values in these two models are similar to those for 3C 184 and 3C 217, and confirm the superiority of the KDA EXT age solution.

\subsubsection{J1428+3938}

$\mathrm{J} 1428+3938$ is a giant-sized $(D>1 \mathrm{Mpc})$ radio galaxy identified as a giant radio galaxy (GRG) by Machalski et al. (2006). Its central radio core that is precisely coincident with a faint optical galaxy with $R=21.11$ mag implies a high redshift; unfortunately, it has not been confirmed spectroscopically. Therefore, the authors estimate its value from the Hubble $m_{R}-z$ relation for giant radio sources as about 0.5. Extended radio lobes of this source have been modelled by Machalski (2011) using the DYNAGE algorithm - contrary to a simpler approach where a dynamical model is fitted to the spectrum of entire source, i.e. to a mean of its two lobes. This is worth emphasizing that such an approach is only possible when a lack of angular resolution does not allow for discrimination between spectra of opposite lobes, especially at low observing frequencies.
Table 4. Flux densities resulting from the KDA and KDA EXT models for 3C 438 and their goodness of fit to the observed data.

\begin{tabular}{rrrr}
\hline \hline$v_{0}$ & $S_{v_{0}} \pm \Delta S_{v_{0}}$ & $\begin{array}{r}S_{\mathrm{MOD}} \\
\text { KDA }\end{array}$ & $\begin{array}{r}S_{\mathrm{MOD}} \\
\text { KDA EXT }\end{array}$ \\
\hline 26 & $228000 \pm 12000$ & 229220 & 243060 \\
38 & $150290 \pm 40650$ & 171455 & 182345 \\
38 & $16284 \pm 48850$ & 171455 & 182345 \\
74 & $81560 \pm 8160$ & 99095 & 105770 \\
86 & $86700 \pm 8720$ & 87287 & 93230 \\
178 & $51400 \pm 4100$ & 46105 & 49377 \\
178 & $49620 \pm 2300$ & 46105 & 49377 \\
325 & $29940 \pm 1080$ & 26506 & 28440 \\
365 & $26400 \pm 950$ & 23766 & 25505 \\
408 & $23760 \pm 480$ & 21387 & 22959 \\
408 & $25180 \pm 1980$ & 21387 & 22959 \\
750 & $13700 \pm 700$ & 11876 & 12727 \\
1400 & $6940 \pm 220$ & 6378 & 6755 \\
1400 & $6854 \pm 220$ & 6378 & 6755 \\
1477 & $6410 \pm 128$ & 6042 & 6388 \\
2695 & $3260 \pm 155$ & 3260 & 3319 \\
2700 & $3300 \pm 160$ & 3259 & 3311 \\
4830 & $1635 \pm 164$ & 1779 & 1638 \\
4850 & $1607 \pm 143$ & 1771 & 1629 \\
4900 & $1580 \pm 60$ & 1752 & 1607 \\
5000 & $1529 \pm 60$ & 1752 & 1565 \\
8440 & $765 \pm 38$ & 986 & 825 \\
10695 & $640 \pm 40$ & 766 & 619 \\
10705 & $600 \pm 38$ & 766 & 619 \\
14900 & $390 \pm 30$ & 538 & 407 \\
\hline$\chi_{\text {red }}^{2}$ & & 8.57 & 1.17 \\
\hline & & &
\end{tabular}

Table 5. Flux densities resulting from the KDA and KDA EXT models for J1428+3938 and their goodness of fit to the observed data

\begin{tabular}{rrrrr}
\hline \hline$v_{0}$ & $S_{v_{0}} \pm \Delta S_{v_{0}}$ & $\begin{array}{r}S_{\mathrm{MOD}} \\
\mathrm{M} 2011\end{array}$ & $\begin{array}{r}S_{\mathrm{MOD}} \\
\text { KDA }\end{array}$ & $\begin{array}{r}S_{\mathrm{MOD}} \\
\text { KDA EXT }\end{array}$ \\
\hline 151 & $971 \pm 109$ & 919 & 995 & 891 \\
151 & $990 \pm 95$ & 919 & 995 & 891 \\
232 & $810 \pm 100$ & 602 & 617 & 582 \\
325 & $428 \pm 34$ & 431 & 421 & 413 \\
408 & $270 \pm 31$ & 344 & 324 & 326 \\
1400 & $83 \pm 4$ & 99 & 73 & 83 \\
4860 & $13 \pm 3$ & 28 & 15 & 15 \\
10550 & $3 \pm 2$ & 13 & 6 & 4 \\
\hline$\chi_{\text {red }}^{2}$ & & 15.52 & 3.69 & 2.20 \\
\hline
\end{tabular}

In order to compare the best determined KDA and KDA EXT model solution to the fit resulting from the Machalski model for the lobes, I use the arbitrary means of $\alpha_{\text {inj }}, t, Q_{\text {jet }}$ and $\rho_{0}$ values given in his Table 2 . These mean values are as follows: $\alpha_{\text {inj }}=0.532, t=415 \mathrm{Myr}, Q_{\text {jet }}=3.93 \times 10^{37} \mathrm{~W}$ and $\rho_{0}=1.46 \times 10^{-22} \mathrm{~kg} / \mathrm{m}^{3}$. In addition to these values, the above model (hereafter M2011) assumed different equations of state, $\Gamma_{\mathrm{c}}=\Gamma_{B}=4 / 3$. 
Table 6. Flux densities resulting from the KDA and KDA EXT models for J1453+3308 and their goodness of fit to the observed data.

\begin{tabular}{rrrrr}
\hline \hline$v_{0}$ & $S_{v_{0}} \pm \Delta S_{v_{0}}$ & $\begin{array}{r}S_{\mathrm{MOD}} \\
\text { MJS2009 }\end{array}$ & $\begin{array}{r}S_{\mathrm{MOD}} \\
\text { KDA }\end{array}$ & $\begin{array}{r}S_{\mathrm{MOD}} \\
\text { KDA EXT }\end{array}$ \\
\hline 151 & $2165 \pm 110$ & 2475 & 2391 & 2399 \\
178 & $2020 \pm 200$ & 2179 & 2136 & 2158 \\
240 & $1667 \pm 250$ & 1715 & 1724 & 1751 \\
325 & $1365 \pm 140$ & 1332 & 1379 & 1378 \\
334 & $1456 \pm 112$ & 1301 & 1351 & 1347 \\
605 & $970 \pm 75$ & 771 & 847 & 843 \\
1287 & $442 \pm 34$ & 389 & 441 & 426 \\
1400 & $426 \pm 26$ & 352 & 410 & 394 \\
4850 & $104 \pm 8$ & 104 & 125 & 115 \\
\hline$\chi_{\text {red }}^{2}$ & \multicolumn{5}{r}{}
\end{tabular}

Table 7. Flux densities resulting from the KDA and KDA EXT models for J1548-3216 and their goodness of fit to the observed data.

\begin{tabular}{rrrrr}
\hline \hline$v_{0}$ & $S_{v_{0}} \pm \Delta S_{v_{0}}$ & $S_{\mathrm{MOD}}$ & $S_{\mathrm{MOD}}$ & $S_{\mathrm{MOD}}$ \\
& & MJK2010 & KDA & KDA EXT \\
\hline 160 & $8400 \pm 840$ & 8174 & 8465 & 8391 \\
334 & $4737 \pm 710$ & 4909 & 4838 & 4975 \\
619 & $3141 \pm 252$ & 3064 & 2983 & 3096 \\
1384 & $1733 \pm 87$ & 1561 & 1544 & 1577 \\
2495 & $963 \pm 30$ & 920 & 928 & 917 \\
4860 & $415 \pm 42$ & 492 & 504 & 476 \\
\hline$\chi_{\text {red }}^{2}$ & & 3.18 & 3.19 & 2.61 \\
\hline
\end{tabular}

It is particularly interesting to examine how the KDA EXT model fits the observed spectrum of this source. I use the $151 \mathrm{MHz}$ measurements as the lowest one and include the very uncertain Miyun measurement at $232 \mathrm{MHz}$. The original radio flux density of $6.2 \mathrm{mJy}$ at $10550 \mathrm{MHz}$ taken from the B3-VLA catalogue (Vigotti et al. 1999), likely referring to the entire source, is corrected here by subtracting the probable flux density of the radio core, supposed to dominate the total radio flux at the high frequencies. This is justified by the $4860 \mathrm{MHz}$ VLA observations showing the core flux of $3.5 \mathrm{mJy}$.

The spectra resulting from the KDA and KDAEXT models and including the data points are shown in Figure 16. The goodness of the fits indicate that the continuum-injection spectra predicted with the M2011 and KDA models are quite comparable, though the fitted values of their free parameters are significantly different. This emphasizes the problem of how to determine the best age solution, reproducing the observed spectrum with a comparable accuracy (again see Brocksopp et al 2011). The KDAEXT model fits the observed data better than KDA. The $\left(t-t_{\mathrm{br}}\right) / t_{\mathrm{br}}$ ratio of about 0.1 (Table 8 ) strongly supports the hypothesis that the observed structure of the lobes and their spectrum reflect the evolutionary phase after the termination of the jet activity.

According to Fig. 16, the flux density measurement at $232 \mathrm{MHz}$ is a clear outlier from the plot and its measurement uncertainty is high. It is reflected in the relatively high values of $\chi_{\text {red }}^{2}$ for the results of modelling. It can be shown that removing this data point from the set reduces the resulting $\chi_{\text {red }}^{2}$ for the KDA EXT model to the value of 1.44. However, in the case of the
KDA solutions it gives the simultaneous increase of this value to about 18.

The most interesting thing here is that if J1428+393 is really at the assumed redshift, it is the old and large source with the evidence of the strong aging in opposition to the two DDRG sources $\mathrm{J} 1453+3308$ and J1548-3216. Furthermore, owing its supposed high redshift, the inverse Compton losses should dominate over synchrotron losses. Indeed, once the jet activity ceases, a strong aging will occur.

\subsubsection{J1453+3308}

$\mathrm{J} 1453+3308$ is the well-known DDRG galaxy whose radio structure has already been studied by several authors (e.g. Schoenmakers et al. 2000; Kaiser et al. 2000; Konar et al. 2006). It consists of two pairs of lobes, the extended outer lobes and slimmer inner ones, considered to be the result of secondary nuclear activity occurring after a termination of the primary jets. Spectral aging analysis of these pairs of lobes was published by Konar et al. (2006), while their dynamical analysis was undertaken by Machalski et al. (2009; in the frame of a subsample of ten GRGs), and, independently by Brocksopp et al. (2011).

Also in this case, the best-fit KDA and KDA EXT flux densities shown in Fig. 17 models were compared to the observed data and to the flux densities of Machalski et al. (2009) model (hereafter MJS2009) for the outer lobes. The arbitrary mean values of the model parameters used for a comparison are $\alpha_{\mathrm{inj}}=0.547$, $t=146 \mathrm{Myr}, Q_{\text {jet }}=4.96 \times 10^{37} \mathrm{~W}$, and $\rho_{0}=2.51 \times 10^{-23} \mathrm{~kg} / \mathrm{m}^{3}$. Additionally, the MJS2009 model assumes $\Gamma_{B}=4 / 3$. The resulting flux densities and the goodness of the fit for each model are given in Table 6 . The values of $\chi_{\text {red }}^{2}$ indicate that the KDA EXT fit is better than MJS2009 and KDA, though the differences in the goodness of the fits are low. These two continuum injection models reproduce well the low-frequency part of the observed spectrum; starting at some point, the KDA EXT spectrum is clearly more bent towards high frequencies. Again, the model spectra predicted with the MJS2009 and KDA models are comparable in spite of the quite different age solutions (reflecting the problem mentioned earlier), and $\chi_{\text {red }}^{2}$ value in the KDA EXT model is similar to that for $\mathrm{J} 1428+3938$. It should be noticed that the models presented here concern the outer (primary) lobes of these DDRGs only. Because the new (secondary) young inner lobes are also observed, it is expected that these outer lobes have had no time for noticeable aging effects.

\subsubsection{J1548-3216}

J1548-3216 (PKS B1545-321) is another example of a giant DDRG. This low-redshift radio galaxy was discovered by Saripalli et al. (2003). Its dynamical analysis was published by Safouris et al. (2008) concluding that the interruption of the jet activity was brief, no more than a small percent of the actual age of the whole source. Nevertheless, based on new low-frequency observations with the GMRT array, Machalski et al. (2010) has repeated the dynamical age analysis but applied to the opposite lobes in both the outer and the inner pairs.

The source location on the southern sky hemisphere means that it is not covered by the large radio surveys on the northern sky: VLSS, NVSS, FIRST, WENSS. The observational data at $150 \mathrm{MHz}$ and $843 \mathrm{MHz}$ from similar southern surveys - TGSS (in progress ${ }^{1}$ ) and SUMSS (Bock et al. 1999), respectively - are

\footnotetext{
1 http://tgssadr.strw. leidenuniv.nl/doku.php
} 
E. Kuligowska: A dynamical model for FR II type radio sources with terminated jet activity

Table 8. Physical parameters of the sources from the sample derived from the best KDA fits for the entire available radio spectrum (upper lines) and KDA EXT fits (bottom lines).

\begin{tabular}{lllllll}
\hline \hline Name & $3 \mathrm{C} 184$ & $3 \mathrm{C} 217$ & $3 \mathrm{C} 438$ & $\mathrm{~J} 1428+3938$ & $\mathrm{~J} 1453+3308$ & $\mathrm{~J} 1548-3216$ \\
\hline$t_{\text {KDA }}[\mathrm{Myr}]$ & 0.78 & 1.87 & 8.5 & 66 & 75 & 55 \\
$t_{\text {KDAEXT }}[\mathrm{Myr}]$ & 0.62 & 1.70 & 13.8 & 158 & 91 & 79 \\
$t_{\text {br }}[\mathrm{Myr}]$ & 0.49 & 1.10 & 12.9 & 145 & 82 & 67 \\
\hline$\alpha_{\text {inj }}$ & 0.58 & 0.59 & 0.61 & 0.84 & 0.59 & 0.64 \\
& 0.55 & 0.53 & 0.54 & 0.58 & 0.51 & 0.60 \\
$Q_{\text {jet }}[\mathrm{W}]$ & $1.3 \times 10^{39}$ & $2.0 \times 10^{39}$ & $2.9 \times 10^{38}$ & $4.1 \times 10^{39}$ & $9.8 \times 10^{37}$ & $8.1 \times 10^{37}$ \\
& $1.6 \times 10^{39}$ & $1.4 \times 10^{39}$ & $2.0 \times 10^{38}$ & $7.1 \times 10^{37}$ & $5.9 \times 10^{37}$ & $4.0 \times 10^{37}$ \\
$\rho_{0}\left[\mathrm{~kg} / \mathrm{m}^{3}\right]$ & $1.2 \times 10^{-22}$ & $3.3 \times 10^{-23}$ & $9.7 \times 10^{-23}$ & $2.2 \times 10^{-23}$ & $7.3 \times 10^{-24}$ & $6.8 \times 10^{-24}$ \\
& $6.3 \times 10^{-23}$ & $1.3 \times 10^{-23}$ & $2.6 \times 10^{-22}$ & $0.68 \times 10^{-22}$ & $0.56 \times 10^{-23}$ & $0.85 \times 10^{-23}$ \\
$p_{\mathrm{c}}\left[\mathrm{N} / \mathrm{m}^{2}\right]$ & $1.3 \times 10^{-9}$ & $7.0 \times 10^{-11}$ & $2.2 \times 10^{-11}$ & $4.1 \times 10^{-13}$ & $4.4 \times 10^{-14}$ & $6.5 \times 10^{-14}$ \\
& $8.4 \times 10^{-10}$ & $3.2 \times 10^{-11}$ & $2.0 \times 10^{-11}$ & $0.89 \times 10^{-13}$ & $2.6 \times 10^{-14}$ & $0.44 \times 10^{-13}$ \\
$U_{\mathrm{c}}[\mathrm{J}]$ & $3.3 \times 10^{52}$ & $2.6 \times 10^{52}$ & $7.8 \times 10^{52}$ & $4.3 \times 10^{54}$ & $2.3 \times 10^{53}$ & $1.4 \times 10^{53}$ \\
& $1.7 \times 10^{52}$ & $6.2 \times 10^{52}$ & $2.2 \times 10^{52}$ & $3.4 \times 10^{53}$ & $2.0 \times 10^{53}$ & $8.7 \times 10^{52}$ \\
$B_{\text {eq }}[\mathrm{nT}]$ & 30 & 7.1 & 4.0 & 0.58 & 0.18 & 0.22 \\
& 24 & 4.8 & 3.7 & 0.25 & 0.13 & 0.18 \\
$v_{\mathrm{h}} / c$ & 0.036 & 0.068 & 0.017 & 0.018 & 0.023 & 0.024 \\
& 0.043 & 0.034 & 0.004 & 0.008 & 0.017 & 0.013 \\
\hline
\end{tabular}

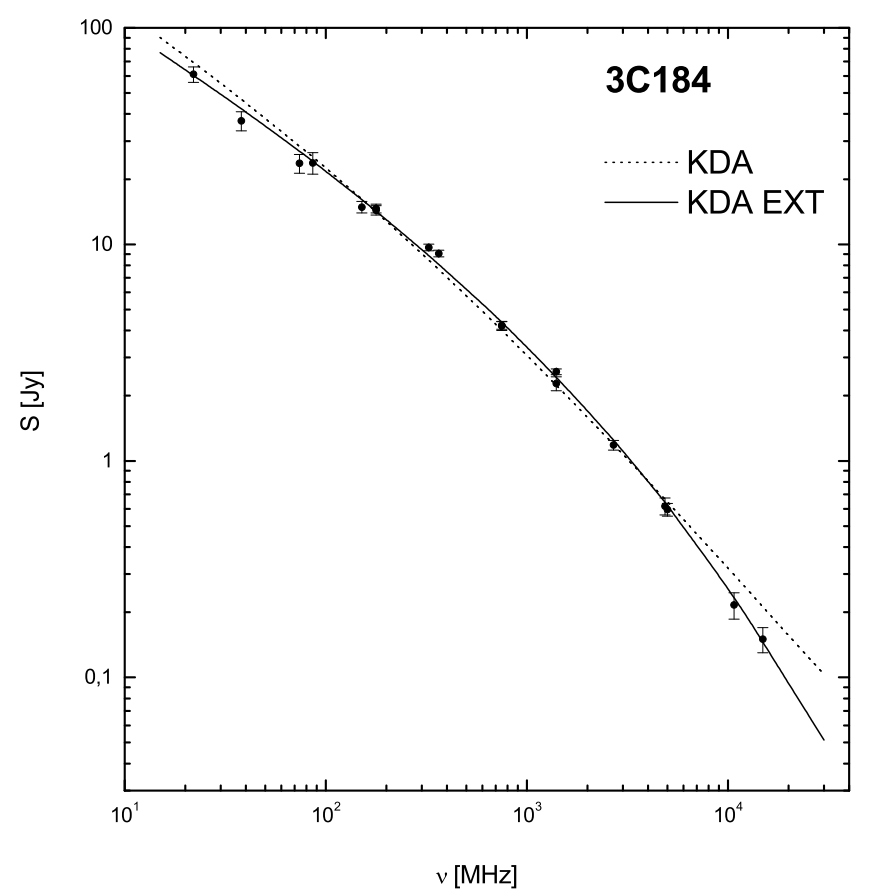

Fig. 13. Best KDA fit (dotted line) and KDA EXT fit (solid line) for the radio galaxy $3 \mathrm{C} 184$. The values of flux density and frequency are presented in logarithmic scale. The observed flux densities and their errors are marked with data points.

not easily available for the source with LAS over 8.7 arcmin on the sky.

In the same way as for $\mathrm{J} 1453+3308$, I calculated the spectra predicted with three models, KDA, KDAEXT, and the Machalski et al. (2010) model (hereafter MJK2010), and compared them to the flux densities measured at six frequencies from $160 \mathrm{MHz}$ up to $4860 \mathrm{MHz}$ adopted from their Table 2. The MJK2010 model for the outer lobes assumes a significant fraction of thermal particles in the lobes $\left(k^{\prime}=10\right)$, thus also

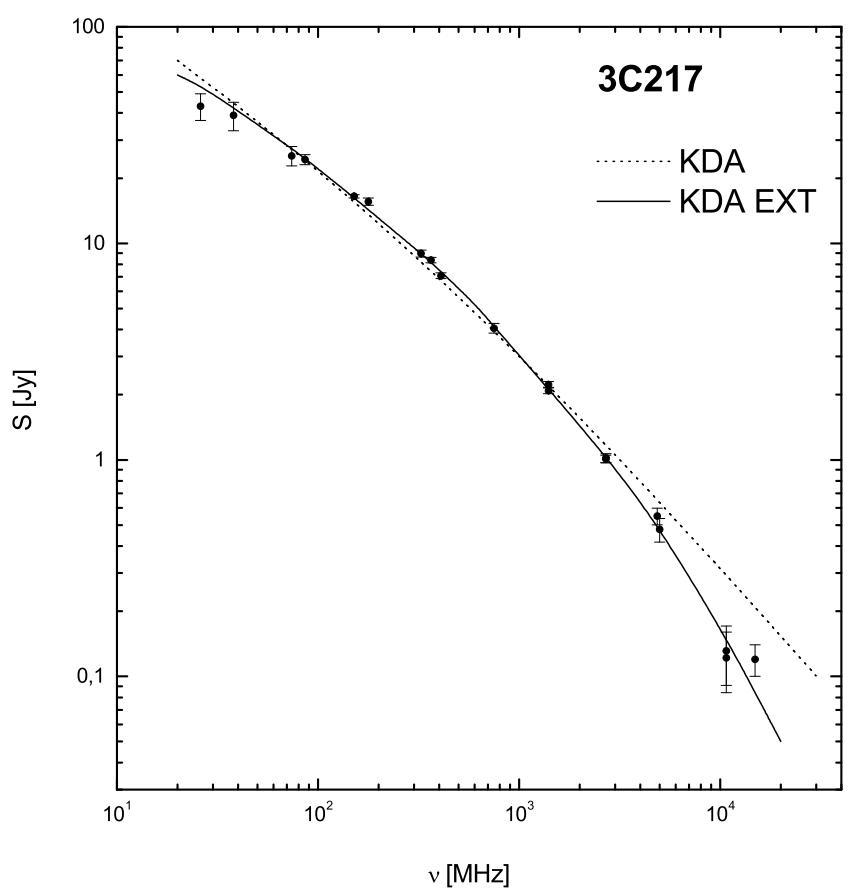

Fig. 14. As in Fig. 13, but for 3C 217.

non-relativistic equations of state, i.e. $\Gamma_{\mathrm{c}}=\Gamma_{B}=\Gamma_{a}=5 / 3$. The arbitrary mean values of the model parameters are taken as $\alpha_{\text {inj }}=0.541, t=132 \mathrm{Myr}, Q_{\text {jet }}=1.12 \times 10^{38} \mathrm{~W}$, and $\rho_{0}=5.55 \times 10^{-23} \mathrm{~kg} / \mathrm{m}^{3}$. The spectra resulting from the predicted flux densities $S_{\text {MOD }}$ (KDA) and $S_{\text {MOD }}$ (KDAEXT) and the goodness of the fit for each model are given in Table 7 and are shown in Fig. 18. Also for this source, the KDA EXT model reproduces the observed spectrum better then the CI models.

It is easily noticeable that in both KDA models (Table 7) the resultant $\chi_{\text {red }}^{2}$ are nearly equal, although the models have significantly different values of their free parameters. This illustrates the problem with the determination of the best KDA solution for 


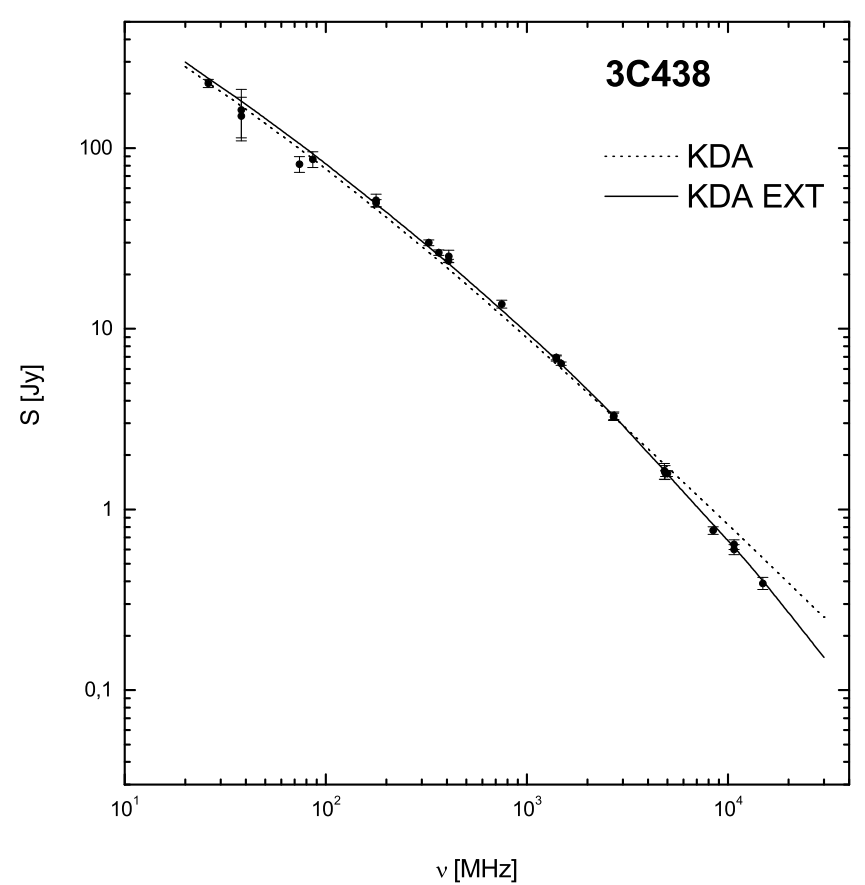

Fig. 15. As in Fig. 13, but for 3C 438.

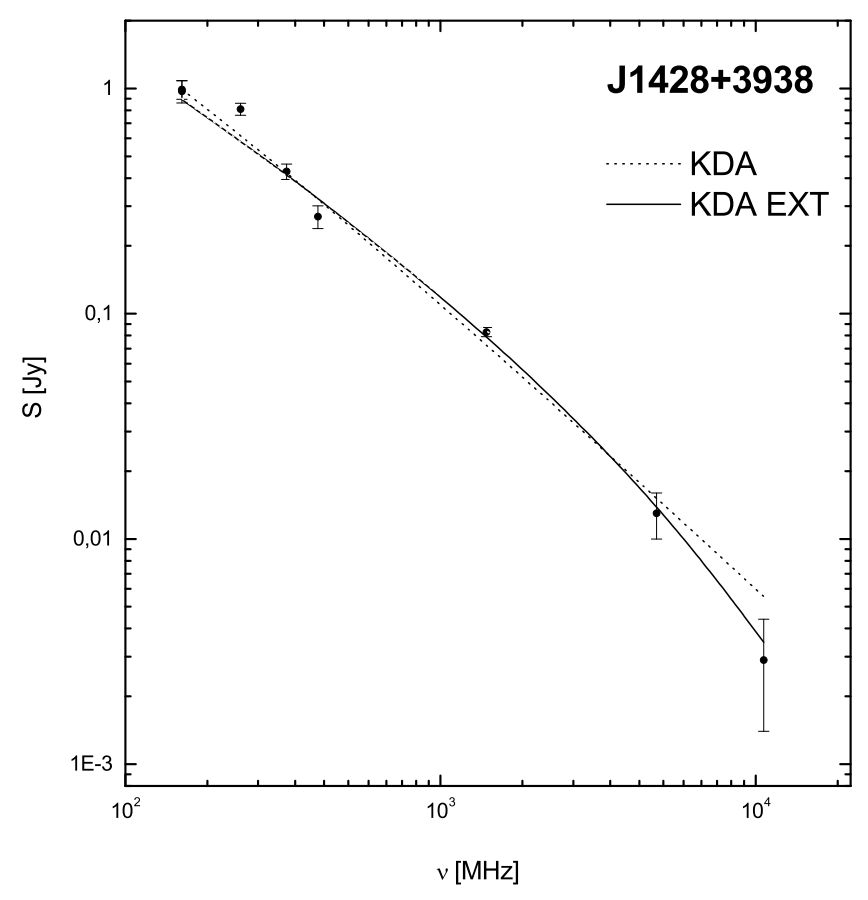

Fig. 16. As in Fig. 13, but for J1428+3938.

a model having a large set of input parameters. The spectra predicted for the fiducial source analysed in Sect. 3.3 clearly show that the shape in a limited frequency range can be very similar (or almost identical). In spite of completely different values of the model parameters (cf. Figs. 11 and 12).

\section{Discussion}

The author is aware that the proposed model of dynamics of the FR-II-type radio sources with retained central activity is based on the widely used original KDA model, which assumes the steady and self-similar flow of energy caused by a supersonic jet.

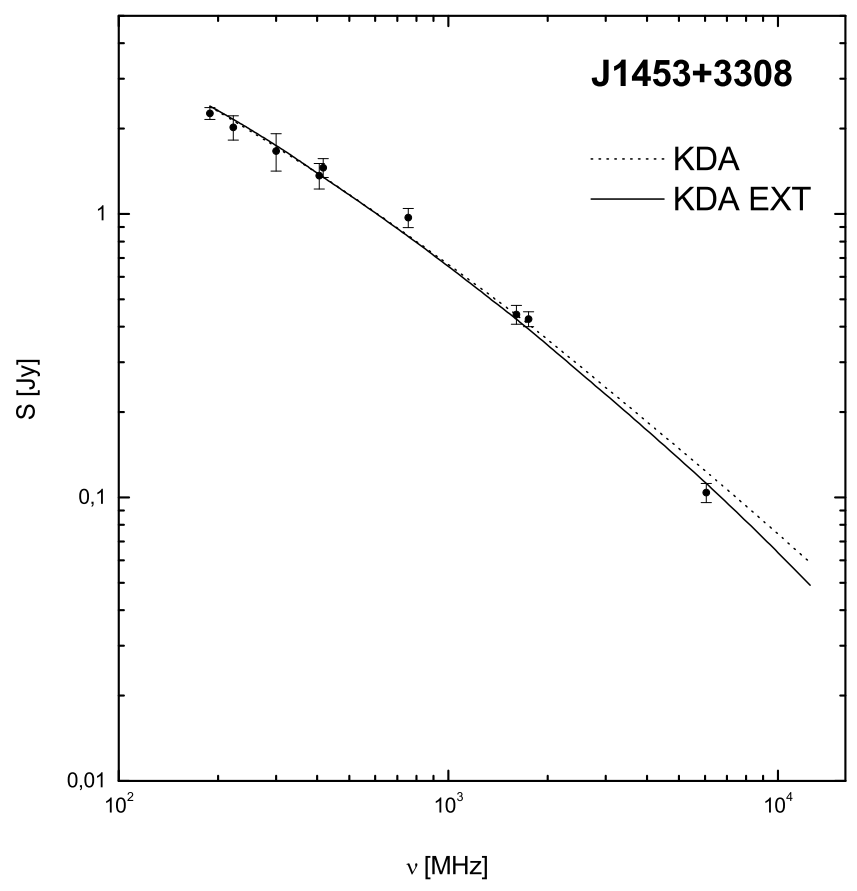

Fig. 17. As in Fig. 13, but for $\mathrm{J} 1453+3308$.

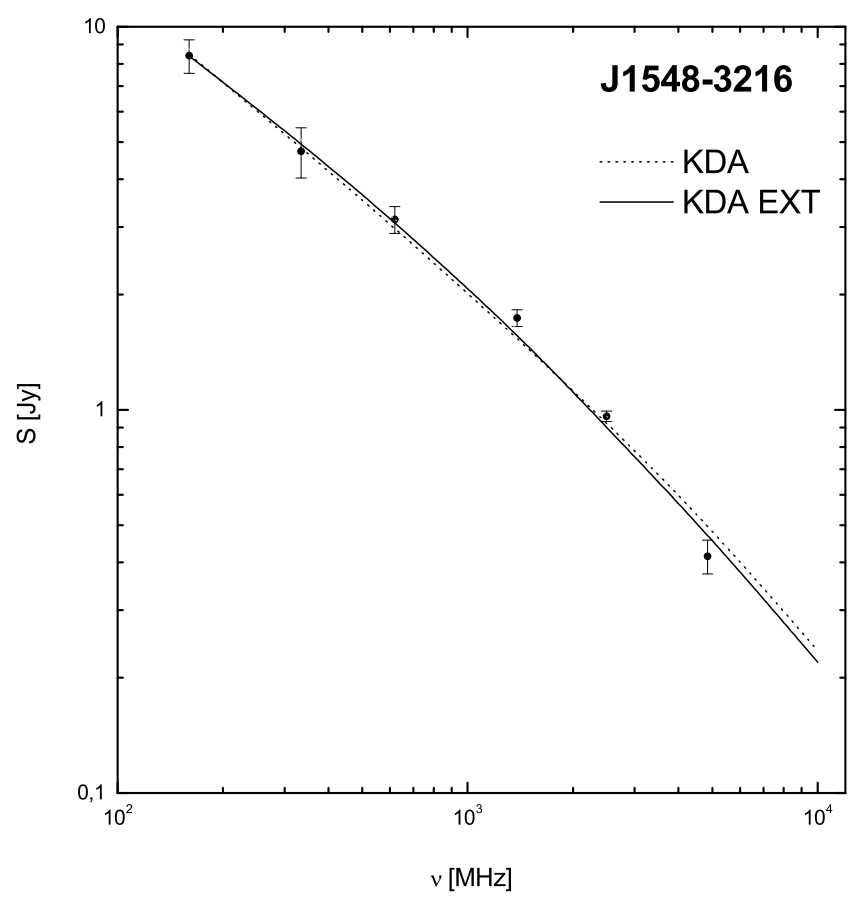

Fig. 18. As in Fig. 13, but for J1548-3216.

This assumption is reasonable in a certain ambient medium density distribution (Falle 1991). If the jet ceases, the self-similarity of the flow appears aimless. Nevertheless, it should be noted that there are numerous sources for which the spectral aging is not yet very strong, i.e. not as evident as in the case of the typical radio relics (Murgia et al. 2011). The solutions and plots presented in Sect. 4 indeed suggest that the KDA EXT model is still useful for those sources whose jets have stopped relatively recently, i.e. the $\left(t-t_{\mathrm{br}}\right) / t_{\mathrm{br}}$ ratios are low (Table 8$)$.

As expected, in the case of two sample DDRGs (J1453+3308 and J1548-3216) the impact of the renewed radio lobes interacting with the old ones is evident and may be responsible for 
relatively limited spectral aging of the source. This translates into the obtained results: the KDA EXT model is not statistically superior in these cases, and its goodness of fit to the observed data is similar to that resulting from the KDA model.

Moreover, I made some attempts to fit the extreme steepened KDA models (with assumed $\alpha_{\text {inj }}$ values in the range of $0.8-1.2$ ) to the observed data for J1428+3309 and two DDRG sources. As a result, most of these fits, although formally best in terms of $\chi_{\text {red }}^{2}$ and rather well reproducing observations, provide very young source ages. However, in some of these solutions evidently nonphysical source parameters were found, i.e. the speed of the lobe expansion significantly greater than the speed of light, $c$. For this reason the obtained results are not included in the plots, and the less steepened solutions (with much lower goodness of fit to the observations) are presented instead. It can then be concluded that for these sources it is not possible to fit a reliable KDA model with very steep spectrum (as could be expected by only taking into account the high-frequency parts of the spectra) because their spectra are both relatively flat at the low frequencies and very steepened at the higher ones. This provides the additional argument for the need to apply the KDA EXT model (as the one that reproduces the real data much better).

It is easy to notice that the KDAEXT models do not satisfactorily reproduce the bent spectra of these sources; there are some observational data points not lying within the model lines, or even favouring the plots of KDA model (cf. Figs. 17 and 18). Other possible reasons for the inconsistency of this model/observations may be a variation in the rate of particles transported in the jets (not implemented in the KDAEXT), or the situation when the jet's activity is not simply stopped, but undergoes a temporary weakening (and then resumes). In addition, the KDAEXT model does not include the possible unknown initial energy distribution in the lobe's head, different from that assumed in the KDA model and demanding a new approach to the understanding of the physics of these sources. However, it needs to be emphasized that the range of available observations for $\mathrm{J} 1428+3938, \mathrm{~J} 1453+3308$, and $\mathrm{J} 1548-3216$ is relatively narrow when compared to the $3 \mathrm{C}$ sources, resulting in not very reliable fits of both models.

It is necessary to take into account the inaccuracy of determining the initial KDA model's parameters obtained for the sample sources and their further use in fitting the KDA EXT model. This may be due to the inaccurate estimation of the initial model parameters for the sources (i.e. the density of the surrounding media, initial particle distributions, or their geometrical parameters). On the other hand, the goodness of the fit to the data obtained for the particular models may be underestimated or overestimated owing to incorrect values of the source's radio flux densities and their errors, which is particularly possible when using observational data from old radio surveys. The application of the KDAEXT model with the prior knowledge of some source parameters (which in this case would be not regarded as its free parameter, but obtained from independent observations), may also improve the accuracy of the solutions. A good example is a possible constraint to the ambient medium density from the modern X-ray observations of the field.

Finally, the $n$-dimensional space of free parameters of the KDA/KDAEXT models is very large and provides multiple solutions (Brocksopp et al. 2011). It appears that the same model outputs may be obtained using very different initial (input) parameters, and very often it is impossible to specify what change in these parameters influences the final solution the most (Sect. 4.3). It may also indicate that the classical approach used to perform the modelling is not accurate enough, and the other numerical methods (general Monte Carlo) should be applied to analyse all the parameters and find the best model fit to the observations.

To sum up, the extended model for the dynamical evolution of the lobes of FR II-type radio galaxies with terminated jet activity, KDA EXT, was briefly presented along with the best fits of this model to the observed radio spectra for the small sample of radio sources. The results were compared to the available KDA solutions for these sources. The preliminary results indicate that KDA EXT model can provide a satisfactory solution in the case of radio sources with presumed large ages or interrupted activity. However, the goodness of its results in relation to the observed radio spectra is supposed to be high only for a certain type of radio sources.

Taking into account all the above, it can be assumed that the KDA EXT model is an appropriate and promising tool for solving the problem of modelling the very steep spectra of FR II radio galaxies, though the accuracy of its solutions is not high and requires further improvements. The future work should focus on the proposed improvements of the KDA EXT model, and on searching for new observational data to perform the further tests of the model.

Acknowledgements. The author express her deeper thanks to the anonymous referee for the useful (and very essential) comments on the manuscript. All the recommendations and remarks significantly contributed to the improvement and further development of this work. I also greatly appreciate the Language Editor's valuable suggestions. This research was supported by the State Committee of Scientific Research through Polish National Science Centre grant No. 2013/09/B/ST9/00599. I sincerely thank professor Jerzy Machalski for carefully reading the manuscript and providing many valuable comments on the article.

\section{References}

Barai, P., \& Wiita, P. J. 2006, MNRAS, 372, 381

Belsole, E., Worrall, D. M., Hardcastle, M. J., \& Birkinshaw, M. 2004, MNRAS, 352,924

Begelman, M., \& Cioffi, D. 1989, ApJ, 345, L21

Blandford, R. D., \& Rees M. J. 1974, MNRAS, 169, 395

Blundell, K. M., Rawlings, S., \& Willot, C. J. 1999, AJ, 117, 677

Bock, D. C. J., Large, M. I., \& Sadler, E. M. 1999, AJ, 117, 1578

Brocksopp, C., Kaiser, C. R., Schoenmakers, A. P., et al. 2011, MNRAS, 410, 484

Carilli, C., Perley, R. A., Dreher, J. W., et al. 1991, ApJ, 383, 554

Cohen, A. S., Lane, W.M., Cotton, W.D., et al., 2007, AJ, 134, 1245

Condon, J. J., Cotton, W. D., Greisen, E. W., et al. 1998, AJ, 115, 1693

Daly, R. A. 1995, ApJ, 454, 580

Douglas, J. N. 1996, VizieR Online Data Catalog, VIII/42

Falle, S. A. E. G. 1991, MNRAS, 250, 581

Fanaroff, B. L., \& Riley, J. M. 1974, MNRAS, 167, 31

Ficarra, A., Grueff, G., \& Tomassetti, G. 1985, A\&AS, 59, 255

Gregory, P. C., Scott, W. K., Douglas, K., \& Condon, J. J. 1996, ApJS, 103, 427

Griffith, M., Heflin, M., \& Conner, S., 1991, ApJS, 75, 801

Hales, S. E. G., Baldwin, J. E., \& Warner, P. J. 1988, MNRAS, 234, 911

Hardcastle, M. J., Alexander, P., Pooley, G. G. et al. 1998, MNRAS, 296, 445

Jaffe, W., \& Perola, G. 1973, A\&A, 26, 423

Kaiser, C. R. 2000, A\&A, 362, 447

Kaiser, C. R., \& Alexander P. 1997, MNRAS, 286, 215

Kaiser, C. R., \& Cotter G. 2002, MNRAS, 336, 649

Kaiser, C. R., Dennett-Thorpe, A., \& Alexander, P. 1997, MNRAS, 292, 723

Kaiser, C. R., Schoenmakers, A. P., \& Rottgering, H. J. A. 2000, MNRAS, 315, 381

Kataoka, J., \& Stawarz, L. 2005, ApJ, 622, 797

Kellermann, K. I., \& Pauliny-Toth, I. I. K. 1973, AJ, 78, 828

King, I. R. 1972, ApJ, 174, 123

Konar, C., Saikia, D. J., Jamrozy, M., et al. 2006, MNRAS, 372, 693

Kühr, H., Nauber, U., Pauliny-Toth, I. I. K., et al. 1979, A Catalogue of radio sources (Bonn: Max-Planck-Institut (MPI) für Radioastronomie)

Kühr, H., Witzel, A., Pauliny-Toth, I. I. K., \& Nauber, U., 1981, A\&AS, 45, 367

Kuligowska E., Jamrozy, M., Kozieł-Wierzbowska, D., et al. 2009, Acta Astron., 59,431

Laing, R. A., \& Peacock, J. A., 1980, MNRAS, 190, 903 
A\&A 598, A93 (2017)

Leahy, J. P., \& Perley, R. A., 1991, ApJ, 102, 2

Machalski, J. 2011, MNRAS, 413, 2429

Machalski, J., Jamrozy, M., Zola, S., et al. 2006, A\&A, 454, 85

Machalski, J., Chyży, K. T., Stawarz, L., et al. 2007, A\&A, 43

Machalski, J., Kozieł-Wierzbowska, D., Jamrozy, M., et al. 2008, ApJ, 679, 149

Machalski, J., Jamrozy, M., \& Saikia, D. J. 2009, MNRAS, 395, 812

Machalski, J., Jamrozy, M., \& Konar, C. 2010, A\&A, 510, A8

Manolakou, K., \& Kirk, J. G., 2002, A\&A 391, 127

Murgia, M., Parma, P., Mack, K.-H., et al. 2011, A\&A, 526, A148

Pacholczyk, A. G., 1970, Radio astrophysics. Nonthermal processes in galactic and extragalactic sources, Series of Books in Astronomy and Astrophysics (San Francisco: Freeman)

Rengelink, R. B., Tang, Y., de Bruyn, A. G., et al. 1997, A\&AS, 124, 259

Riley, J. M. W., Waldram, E. M., \& Riley, J. M. 1999, MNRAS, 306, 31
Roger, R. S.; Costain, C. H., \& Stewart, D. I. 1986, A\&AS, 65, 485

Safouris, V., Subrahmanyan, R., Bicknell, G., et al. 2008, MNRAS, 385, 2117

Saikia, D. J., Jamrozy, M., Konar, C., et al. 2010, Proc. 25th Texas Symp. on Relativistic Astrophysics, December 6-10, Heidelberg, 14

Saripalli, L., Subrahmanyan, R., \& Udaya Shankar, N. 2003, ApJ, 590, 181 Scheuer, P. A. G. 1974, MNRAS, 166, 513

Schoenmakers, A. P., de Bruyn, A. G., Rottgering, H. J. A., et al. 2000, MNRAS, 315,371

Shklovskii, I. S., 1963, SvA, 6, 465

Vigotti M., Gregorini, L., Klein, U., et al. 1999, A\&AS, 139, 359

Waldram, E. M., Yates, J. A., Riley, J. M., et al. 1996, MNRAS, 282, 779

White, R., \& Becker R. H., 1992, ApJ, 79, 331

Wright, E. 2006, PASP, 118, 1711

Zhang, X., Zheng, Y., Chen, H., et al. 1997, A\&AS, 121, 59 